\title{
Tabularia
}

\section{Comment les Satires de Juvénal sont arrivées au Mont Saint-Michel ? (enquête sur Paris, BnF, lat. 8070-I et sur son modèle)}

How Did the Satires of Juvenal Arrive at the Mont Saint-Michel? (Study of Paris, BnF, lat. 8070-I and Its Model)

Come sono arrivate le Satire di Giovenale a Mont Saint-Michel? (indagine su Paris, BnF, lat. 8070-I e sul suo modello)

Wie sind die Satiren von Juvenal in Mont Saint-Michel angekommen? Eine Handschriftenuntersuchung zu Paris, BnF, lat. 8070-I und über ihre Vorlage

\section{Frédéric Duplessis}

\section{OpenEdition \\ Journals}

Édition électronique

URL : https://journals.openedition.org/tabularia/5128

DOI : 10.4000/tabularia.5128

ISSN : $1630-7364$

Éditeur :

CRAHAM - Centre Michel de Boüard, Presses universitaires de Caen

\section{Référence électronique}

Frédéric Duplessis, «Comment les Satires de Juvénal sont arrivées au Mont Saint-Michel ? (enquête sur Paris, BnF, lat. 8070-l et sur son modèle) », Tabularia [En ligne], Autour de la Bibliothèque virtuelle du Mont Saint-Michel, mis en ligne le 25 mai 2021, consulté le 27 mai 2021. URL : http:// journals.openedition.org/tabularia/5128; DOI : https://doi.org/10.4000/tabularia.5128

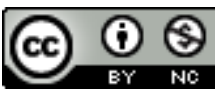

la revue Tabularia est mise à disposition selon les termes de la Licence Creative Commons Attribution - Pas d'Utilisation Commerciale 4.0 International. 

Comment les Satires de Juvénal sont arrivées au Mont Saint-Michel? (enquête sur Paris, BnF, lat. 8070-I et sur son modèle)

How Did the Satires of Juvenal

Arrive at the Mont Saint-Michel?

(Study of Paris, BnF, lat. 807o-I and Its Model)

Come sono arrivate le Satire di Giovenale

a Mont Saint-Michel?

(indagine su Paris, BnF, lat. 8o7o-I e sul suo modello)

Wie sind die Satiren von Juvenal in Mont Saint-Michel

angekommen? Eine Handschriftenuntersuchung

$z u$ Paris, BnF, lat. 8070-I und über ihre Vorlage

Frédéric DUPLESSIS

ENS de Lyon, CIHAM

frederic.duplessis@ens-lyon.fr

Résumé:

L'analyse du contenu de la première unité codicologique du manuscrit Paris, BnF, lat. 8070 nous permet d'étudier la façon dont était lu Juvénal au Mont Saint-Michel peu après l'arrivée des bénédictins aux alentours de l'an Mil. Le texte des Satires et leurs gloses montrent que la réception montoise du satiriste est influencée par l'enseignement de Remi d'Auxerre et que le modèle utilisé par les moines normands provient sans doute d'une bibliothèque du nord-est de la France. L'étude des textes scolaires copiés après les Satires dans ce manuscrit (fol. 124-128) confirme ce lien avec les écoles du nord-est de la France et nous invite à supposer l'existence d'un réseau d'échanges intellectuels reliant le Mont à la région de Reims par l'intermédiaire de Fleury ou de Corbie.

Mots-clés: Juvénal, gloses, écoles carolingiennes, réseaux intellectuels, trivium

Abstract:

The analysis of the content of the first codicological unit of the manuscript Paris, BnF, lat. 8070 allows us to study how Juvenal was read at Mont Saint-Michel shortly after the arrival of the Benedictines around the year 1000. The text of the Satires and their glosses show that the reception of the satirist at Mont Saint-Michel is influenced by the teaching of Remigius of Auxerre and that the model used by the Norman monks probably comes from a library in north-eastern France. The study of the school texts copied after the Satires in this manuscript (fol. 124-128) corroborates this link with schools of north-eastern France and we may assume the existence of a network of intellectual exchanges linking the Mont to the Reims region through Fleury or Corbie.

Keywords: Juvenal, glosses, carolingian schools, intellectual networks, trivium

Tabularia "Études", "Autour de la Bibliothèque virtuelle...", 2021, p. 1-30, 25 mai 2021 URL: http://journals.openedition.org/tabularia/5128 | DOI: 10.4000/tabularia.5128 
Riassunto:

L'esame dei testi trasmessi nella prima unità codicologica del manoscritto Paris, BnF, latin 8070 ci permette di studiare il modo in cui Giovenale era letto a Mont Saint-Michel, verso l'anno Mille, poco dopo l'arrivo dei monaci benedettini. Le Satire e le loro glosse mostrano che la loro ricezione a Mont Saint-Michel è influenzata dall'insegnamento di Remigio d'Auxerre e che il modello utilizzato dai monaci normanni proviene da una biblioteca del Nord-Est della Francia. Lo studio dei testi ad uso scolastico copiati dopo il testo delle Satire (fol. 124-128) conferma questo legame con le scuole del Nord-Est della Francia e ci invita a ipotizzare l'esistenza di una rete di scambi intellettuali che legarono Mont Saint-Michel alla regione di Reims grazie alla mediazione di Fleury o di Corbie.

Parole chiave: Giovenale, glosse, scuole carolingie, reti intellettuali, trivium

Abstrakt:

Die Analyse des Inhalts der ersten kodikologischen Einheit der Handschrft Paris, BnF, lat. 8070 ermöglicht uns zu untersuchen, auf welche Art Juvenal in Mont St-Michel kurz nach der Ankunft der Benediktiner um das Jahr 1000 gelesen wurde. Der Text der Satiren und die dazugehörigen Glossen zeigen, dass die Rezeption des Satirikers in Mont St-Michel von dem Bildungswesen Saint-Remis von Auxerre beeinflusst wurde und dass die Vorlage, die von den normannischen Mönchen verwendet wurde, zweifellos aus einer Bibliothek aus dem Nordosten von Frankreich stammt. Die Beschäftigung mit den Texten, die in der Handschrift aufdie Satiren folgen (fol. 124-128) bestätigt diese Verbindung zu den Schulen im Nordosten Frankreichs und veranlasst uns dazu die Existenz eines intellektuellen Tauschnetzwerkes anzunehmen, das Mont St-Michel mit der Region von Reims über die Vermittlung von Fleury oder Corbie verband.

Schlagworte: Juvenal, Glossen, karolingische Schulen, Gelehrtennetzwerke, Trivium

Nous n'avons conservé qu'un petit nombre de manuscrits de textes classiques copiés en Normandie avant le XII ${ }^{e}$ siècle ${ }^{1}$. Parmi ces rares exemplaires, il est intéressant de remarquer que l'on trouve cinq manuscrits copiés au Mont SaintMichel à la fin du Xe et au début du XI ${ }^{e}$ siècle (autour de l'abbatiat de Mainard II):

- Avranches, Bibl. mun., 229, fol. 116-190 (divers ouvrages de Boèce);

- Avranches, Bibl. mun., 240 (De Nuptiis Philologiae et Mercurii de Martianus Capella ${ }^{2}$;

- Paris, BnF, Lat. 8055-II, fol. 141-178 (Satires de Perse et énigmes de Symphosius);

- Paris, BnF, Lat. 8070-I, fol. 1-128 (Satires de Juvénal);

- Vaticano, BAV, Reg. lat. 2043 (Saturnales de Macrobe).

1. Ce travail a bénéficié des relectures de Jérémy Delmulle et de Stéphane Lecouteux, que je remercie pour toutes leurs suggestions.

2. Sur ce manuscrit, voir la contribution de J.-B. Guillaumin à paraitre dans le présent dossier de Tabularia. 
La copie de ces manuscrits est une conséquence de la reprise en main de l'abbaye par les bénédictins à partir de 966. Elle se traduit par la constitution rapide d'une bibliothèque monastique au Mont Saint-Michel dès la fin du X $\mathrm{X}^{\mathrm{e}}$ siècle et l'on peut constater que les classiques figurent en bonne place au sein des textes que les premiers bénédictins montois ont jugé utiles de posséder dans leur abbaye.

Les travaux de François Avril et de Jonathan J. Alexander ont permis de retrouver plus d'une dizaine de volumes copiés durant ces années 990-1015³. L'étude de ce fonds homogène nous donne accès à la vie intellectuelle au Mont Saint-Michel autour de l'an Mil et nous permet de voir quels textes étaient lus à cette époque et comment ils étaient lus. La bonne conservation de cette strate nous invite aussi à rechercher les bibliothèques à l'origine de ce premier fonds bénédictin et ainsi à reconstituer le réseau intellectuel dans lequel s'insérait l'abbaye à cette époque. Ce sont ces enquêtes que je vais tenter de mener en étudiant la première unité codicologique d'un de ces volumes, le manuscrit Paris, BnF, lat. 8070.

\section{Présentation du volume}

Ce manuscrit de Juvénal fait partie des volumes ayant quitté l'abbaye du Mont entre le début des guerres de Religion et l'installation de la congrégation de SaintMaur en 1622. Il a appartenu à l'historien et écrivain Claude Fauchet (1530-1602) avant d'entrer plus tard dans la bibliothèque de Jean-Baptiste Colbert puis dans celle du roi. Le volume est aujourd'hui composé de deux unités codicologiques. La première provient du Mont. Elle occupe les fol. 1-128 et a été copiée dans l'abbaye normande à la fin du $\mathrm{X}^{\mathrm{e}}$ ou au début du XI ${ }^{\mathrm{e}}$ siècle. La seconde unité codicologique correspond aux actuels fol. 131r-142r (les feuillets 129 et 130 manquent dans la foliotation). Copiée au cours du XI ${ }^{\mathrm{e}}$ siècle en Italie du nord ou dans le sud de la France, elle contient principalement les Satires de Perse avec quelques gloses. Voici le contenu détaillé de la première unité codicologique:

- fol. 1-16v: une série de glossae collectae portant sur le début de l'œuvre de Juvénal (Sat. I, 1 - VI, 164);

- fol. 17r-124r: un accessus carolingien sur Juvénal suivi des seize Satires accompagnées de nombreuses gloses contemporaines interlinéaires et marginales;

- fol. 124r-126r: une lettre érudite sur le terme juvénalien ceroma que ses éditeurs modernes nomment Quid sit ceroma;

- fol. 126r-127r: une généalogie d'Énée et de Priam;

- fol. 127v-128r: des extraits d'Augustin et de Grégoire, suivis de notes scolaires sur la dialectique et la philosophie;

- fol. 128v: du matériel lexicographique (deux differentiae et un petit glossaire gréco-latin).

3. Avril, 1967; Alexander, 1970, notamment p. 24-26 et 212. 
Cette unité codicologique est constituée de seize quaternions réguliers (sans signatures visibles), copiés par quatre scribes. La main principale (A) a copié le texte des Satires ainsi que Quid sit ceroma et la généalogie d'Énée. Elle se caractérise, notamment, par l'utilisation de quatre graphies distinctes pour la lettre $a$. La deuxième main la plus importante (B) a copié la série de gloses sur les deux premiers cahiers et la majorité des gloses marginales dans les cahiers suivants. La troisième et la quatrième mains $(\mathrm{C}$ et $\mathrm{D})$ ont complété les feuillets restés vides à la fin du seizième cahier (fol. 127v-128r pour C, peut-être en deux temps, et fol. $128 \mathrm{v}$ pour D) en ajoutant des textes variés sans lien apparent avec Juvénal. L'analyse paléographique recoupe donc l'étude du contenu de cette unité codicologique: il s'agit d'un exemplaire de Juvénal homogène jusqu'au fol. 127r, avec plusieurs additions contemporaines ajoutées par deux autres mains aux fol. 127v-128r.

Ce manuscrit a été rattaché à l'atelier d'écriture du Mont Saint-Michel grâce aux analyses paléographiques de François Avril ${ }^{4}$, qui a reconnu la main principale de ce manuscrit dans plusieurs autres manuscrits provenant du Mont SaintMichel et a proposé de l'identifier avec un certain Hervardus dont le nom est donné par un colophon contenu dans le manuscrit Leiden, UB, Voss. lat. F 39, fol. $135 \mathrm{v}$ (Fratris Heruardi post longum penna laborem optatam gaudens hic tenuit requiem $\left.{ }^{5}\right)^{6}$. Cette main a, notamment, copié une liste de moines du Mont SaintMichel sous l'abbatiat de Mainard II (991-1009). Cette liste ajoutée dans un manuscrit passé par Fleury-sur-Loire et aujourd'hui conservé à Orléans ${ }^{7}$ nous permet de dater de la fin du $\mathrm{X}^{\mathrm{e}}$ ou du début du XI ${ }^{\mathrm{e}}$ siècle l'ensemble des manuscrits où l'on rencontre cette main. Par ailleurs, je signale que la main $B$ se retrouve au début du Macrobe copié au Mont à la même époque (actuel Vaticano, BAV, Reg. lat. 2043) et que la main $C$ est proche de la troisième main du manuscrit montois de Martianus Capella (Avranches, Bibl. mun., 240, fol. 98-101).

\section{Le texte de Juvénal et son commentaire}

Ce manuscrit contient l'ensemble des Satires de Juvénal copiées à raison de dix-huit vers par page. À bien des égards, il s'agit d'un exemplaire juvénalien typique des écoles françaises de l'époque post-carolingienne. Les Satires y sont divisées en cinq livres. Le texte de ce manuscrit correspond à un état textuel déjà très corrompu largement répandu, qualifié de «vulgate» par les éditeurs ${ }^{8}$. Je donnerai simplement deux exemples de corruptions. La fameuse formule panem et circenses (Sat. X, 81) est transmise sous la forme pan et circenses, éminemment plus répandue durant l'époque médiévale ${ }^{9}$. La bonne leçon, panem, n'est signalée que comme une variante notée dans la marge du fol. 84r («Aliter panem»).

\footnotetext{
4. Alexander 1967, 242.

5. Il s'agit d'un distique élégiaque avec rime.

6. Sur Heruardus, voir AvrIL, 1967, 204-205 et AleXANDER, 1970, 39.

7. Orléans, Bibl. mun., 105, p. 361.

8. Sur la transmission du texte de Juvénal, voir TARRANT, 1983.

9. Cf. SANDFORD, 1951
} 


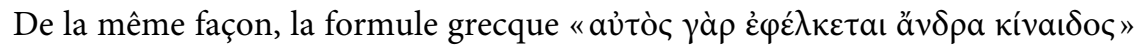
(Sat. IX, 37), qui parodie une tournure homérique ${ }^{10}$, est copiée ainsi dans le manuscrit du Mont: «AITOC $\Gamma \Lambda$ IKOY AN $\triangle \mathrm{PA}$ KINAI $\Delta \mathrm{OC}$ ». Cette leçon, bien qu'intraduisible, se retrouve dans la majorité des manuscrits de Juvénal. Ces deux corruptions ne seront d'ailleurs définitivement résolues qu'avec l'édition de Pierre Pithou de 1585, fondée sur la redécouverte du meilleur témoin des Satires, l'actuel manuscrit Montpellier, BIUM, H 125.

Au sein de la vulgate des manuscrits juvénaliens $(\Omega)$, U. Knoche rattache le texte transmis par le manuscrit du Mont à la branche $\psi$ de la tradition textuelle de Juvénal ${ }^{11}$, qui serait composée, selon lui, de cinq témoins:

- Leiden, UB, VLF 64 (X', Auxerre/Reims);

- Leiden, UB, VLQ 18 (X², Auxerre/ Reims);

- Paris, BnF, lat. 4883a $\left(\mathrm{XI}^{\text {in }}\right.$, Limoges $\left.{ }^{12}\right)$;

- Paris, BnF, lat. 8070 (X-XI, Mont Saint-Michel);

- Paris, BnF, lat. 8072, fol. 1-47 (X, France? provenance: Lagny-sur-Marne, puis Pierre Pithou).

D'après U. Knoche, les trois manuscrits de Paris ont le même ancêtre que le modèle des deux manuscrits de Leiden.

Dans le manuscrit du Mont, le texte de Juvénal est accompagné de nombreuses gloses et annotations. Là aussi, cela n'a rien d'original pour un texte scolaire et difficile comme les Satires. On rencontre, par exemple, plusieurs nota marginaux dans le manuscrit Paris, BnF, lat. 8070 pour mettre en valeur un élément du texte. Si cette pratique est courante, il est intéressant de noter que la forme du nota est plus originale (avec la lettre "o" copiée en bas à droite sous la barre du " $\mathrm{t}$ " et la lettre "a" ouverte tracée juste au-dessus de la barre verticale du " $\mathrm{t}$ " de manière à en former la barre horizontale $)^{13}$. Cette graphie de nota se retrouve dans plusieurs autres manuscrits scolaires copiés au Mont à la même époque ${ }^{14}$.

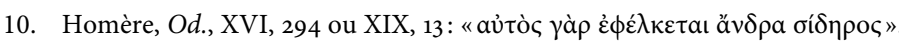

11. KNOCHE, 1940, $181,188-189$ et 378 .

12. Les textes copiés aux fol. 1-66r de ce manuscrit se retrouvent tous dans le manuscrit Vaticano, $\mathrm{BAV}$, Reg. lat. 215 (copié vers 877, peut-être à Laon), qui a sans doute servi de modèle pour réaliser cette partie du manuscrit. Ces deux manuscrits transmettent du matériel scolaire qui circulaient dans les écoles de la région de Reims.

13. Ce nota est utilisé dans les marges des fol. 20r, 21rv, 27v, 32r, 33rv, 34v, 35rv, 4or, 49r, 5or, 57r, 59r, 61v, 85v, 87r, 88v, 9or, 91r, 92r-v, 95rv, 97v, 98rv, 104r-v, 105r, 108r-v, 111v, 112r, 115v, 116r, 125r-v; on trouve une version légèrement différente (sans le 'o') aux fol. $32 \mathrm{r}, 36 \mathrm{v}, 37 \mathrm{r}$, $113 \mathrm{r}$.

14. Je l'ai retrouvée dans les manuscrits montois de Martianus Capella (Avranches, Bibl. mun., 240, fol. 31v), de Macrobe (Vaticano, BAV, Reg. lat. 2043, fol. 88r), de Boèce (Avranches, Bibl. mun., 229, fol. 84v) ou de Perse (Paris, BnF, lat. 8055, fol. 149v). Cette liste n'est probablement pas exhaustive. Dans le manuscrit de Perse, nota est souvent accompagné de l'abréviation «dł» (pour «dignum legendi»?), qui est beaucoup plus rare. Evina Steinová, spécialiste des notes marginales carolingiennes, m'a confirmé qu'elle ne l'avait jamais rencontrée. Cette combinaison se retrouve au fol. 104r du manuscrit de Juvénal. L'association de l'abréviation «DM» (pour «dignum memoriae») avec un nota est plus fréquente (pour les manuscrits de Juvénal, voir, par exemple, Cambridge, King's College, 52, fol. 17r). 
Les gloses marginales et interlinéaires qui accompagnent le texte de Juvénal appartiennent au commentaire le plus répandu à l'époque, celui composé par Remi d'Auxerre à la fin du IX $\mathrm{IX}^{\mathrm{e}}$ siècle sans doute à Auxerre et peut-être aussi à Reims, que les éditeurs modernes nomment Scholia recentiora pour les distinguer des scholies tardo-antiques ${ }^{15}$. Ces gloses carolingiennes se sont diffusées sous trois formes: une branche $\varphi$, liée principalement à l'espace français et dont le manuscrit montois est un des témoins, une branche $\chi$, diffusée avant tout en Suisse, en Lombardie et dans l'ouest de l'Allemagne, et une branche contaminée. Voici les principaux témoins de ces Scholia recentiora sur Juvénal:

\section{Rédaction $\varphi$}

- V: Leiden, UB, Voss. lat. Q 18 ( $\mathrm{X}^{2}$, Auxerre/Reims);

- W: Wien, ÖNB, 131 (X-XI, Hersfeld);

- D: Paris, BnF, lat. 8070 (X-XI, Mont Saint-Michel);

- B: Leiden, UB, Voss. lat. F 64 (X², Auxerre/Reims).

\section{Rédaction $\chi$}

- U: Vaticano, BAV, Urb. lat. 661 (XI, Allemagne ou nord de l'Italie?);

- H: Paris, BnF, lat. 9345 (X ${ }^{\text {ex }}$, Echternach ou Trèves);

- A: München, BSB, Clm 408 (XI, Allemagne);

- T: Vaticano, BAV, Vat. lat. 2810 (X, nord de l'Italie);

- Y: Einsiedeln, Stiftsbibl., 34 (X, Einsiedeln);

- X: St. Gallen, Stiftsbibl., 871 (XI, Saint-Gall);

- E: London, BL, Add. 30861 (XI, Wurtzbourg, puis Constance).

\section{«Testimoni misti» (signalés par S. Grazzini mais non utilisés)}

- Firenze, Laurenziana, Plut. 34. 42 (XI, Italie);

- Vaticano, BAV, Urb. lat. 342 (X'1, Echternach);

- Wien, ÖNB, 277 (X, France?);

- Vaticano, BAV, Pal. lat. 1701 (X², Allemagne);

- Wolfenbüttel, HAB, Gud. lat. 156 (XI);

- Bruxelles, BR, 9973 (XI, Allemagne);

- Vaticano, BAV, Reg. lat. 2029 (XI, France?);

- Firenze, Laurenziana, San Marco 234 (XI-XII, Italie).

15. Ces gloses ont fait l'objet d'une édition récente en deux volumes: GrazZINI, 2011 et GrazZINI, 2018. 
Le contenu de cette strate exégétique carolingienne est avant tout explicatif: on y trouve de nombreuses reformulations et explicitations, ainsi que des définitions souvent accompagnées d'étymologies. Mais, à côté de ce matériel, une partie importante des Scholia recentiora (notamment les gloses marginales) a une visée encyclopédique. Ces gloses renferment, par exemple, des développements historiques, mythologiques, géographiques ou scientifiques.

Par ailleurs, dans les manuscrits juvénaliens du $\mathrm{X}^{\mathrm{e}}$ et du $\mathrm{XI}^{\mathrm{e}}$ siècle, il n'est pas rare de rencontrer, en guise d'introduction, des vies de Juvénal accompagnées de quelques gloses portant notamment sur les noms de Juvénal. Dans le manuscrit du Mont, on trouve ce genre de proto-accessus au premier feuillet et au fol. 17r-v. Leur contenu rapproche le manuscrit Paris, $\mathrm{BnF}$, lat. 8070 de trois autres manuscrits ${ }^{16}$ :

- Leiden, UB, VLF 64, fol. 1v-2r ( $\mathrm{X}^{2}$, Auxerre-Reims);

- Paris, BnF, lat. 4883a, fol. 67r-v (XI ${ }^{\text {in }}$ Limoges);

- Wien, ÖNB, 277 (X, France?).

J'ai insisté jusqu'ici sur les caractéristiques que le manuscrit du Mont partage avec la majorité des manuscrits de Juvénal de l'époque post-carolingienne. Il convient désormais de souligner deux particularités plus originales. La première concerne la division du texte des Satires en paragraphes au moyen de gammas. Si le recours à ce signe graphique pour faire apparaître des unités thématiques n'est pas rarissime dans les manuscrits d'auteurs classiques ${ }^{17}$, on en conserve peu d'exemples pour les manuscrits de Juvénal ${ }^{18}$. En revanche, cette pratique se retrouve dans plusieurs manuscrits copiés au Mont Saint-Michel à la même époque, notamment dans les manuscrits de Martianus et de Macrobe (Avranches, Bibl. mun., 240 et Vaticano, BAV, Reg. lat. 2043) et dans celui de Perse (Paris, BnF, lat. 8055), où le gamma est parfois accompagné de l'indication marginale Aliud thema (fol. 149r).

Par ailleurs, le Juvénal du Mont se distingue aussi par la volonté qu'ont eue ses concepteurs de constituer un commentaire lemmatique continu à partir des gloses marginales de Remi d'Auxerre, pour fournir aux lecteurs des Satires l'équivalent des commentaires de Servius sur Virgile. Ce genre de tentative visant à rassembler le matériel marginal en un commentaire séparé du texte se rencontre dans trois autres manuscrits des Scholia recentiora ${ }^{19}$, mais le manuscrit Paris, BnF, lat. 8070 est le seul témoin de la branche $\varphi$ des Scholia recentiora à présenter cette caractéristique.

16. Sur ces proto-accessus sur Juvénal, je me permets de renvoyer à mon édition (dans Grazzini, 2018, 332-347) et à mon étude (Duplessis, 2017).

17. Cf. Munk Olsen, 2009, 257.

18. J'ai consulté une vingtaine de manuscrits antérieurs au XII siècle et je n'ai trouvé que deux autres exemples où le texte de Juvénal comporte de nombreuses marques de division contemporaines de la copie: Cambridge, Trinity College, O.4.10 (1241) (X, Canterbury) et London, Royal 15 B XII $\left(\mathrm{X}^{1}\right.$, centre de la France). Dans le manuscrit de Cambridge, la capitulation est marquée avec des $\mathrm{K}$, tandis que le manuscrit de Londres utilise des gammas comme celui de Paris.

19. On trouve, en effet, des tentatives équivalentes dans deux manuscrits de la famille $\chi(U$ et $Y)$ et dans le manuscrit contaminé Wien, ÖNB, 277. Sur $U$, voir GrazzinI, 2011, xx-xxI; sur $Y$, où la tentative est avortée dès Sat. I, 117, voir DupLessis, 2017, 110, n. 23; sur le manuscrit de Vienne, je renvoie à ma description dans GraZZINI, 2018, 339-340. 
Pour réaliser ce projet, la copie du manuscrit a été faite par deux copistes qui travaillaient en étroite collaboration probablement à partir d'un antigraphe dérelié: le scribe A copiait le texte de Juvénal et, au fur et à mesure qu'il progressait, il confiait à son collègue, le scribe $\mathrm{B}$, les cahiers déjà transcrits pour que celui-ci copie de son côté sous forme de glossae collectae les gloses marginales présentes dans ces cahiers. Ce mode de fonctionnement explique pourquoi les deux premiers cahiers sont copiés par la main qui copie uniquement les gloses marginales dans les cahiers suivants. On constate que ce processus a finalement été abandonné: le commentaire continu s'arrête, en effet, à la fin du deuxième cahier au milieu d'une glose portant sur Sat. VI, 164, c'est-à-dire exactement à l'endroit où on voit réapparaître les gloses marginales dans les marges du texte de Juvénal (au fol. 49v) ${ }^{20}$.

L'abandon de ce projet s'explique probablement par son caractère extrêmement fastidieux, reflété par plusieurs accidents plus ou moins importants concernant les gloses portant sur le début des Satires (jusqu'en VI, 164). On retrouve ainsi des gloses copiées deux fois ${ }^{21}$ ou, à l'inverse, des gloses d'abord oubliées et ajoutées dans un second temps ${ }^{22}$. L'erreur la plus conséquente est sans aucun doute l'inversion du contenu des fol. $4 \mathrm{v}$ et $5 \mathrm{r}$, signalée par une note copiée dans la marge du fol. $4 \mathrm{v}$ («tota haec sinistra pagina post sequentem dexteram legenda est») ${ }^{23}$. Toutes ces difficultés expliquent l'abandon de cette tentative. Dès que le scribe principal termina son travail avec les Satires, le scribe secondaire se contenta d'achever le deuxième cahier et décida de recopier le reste des gloses marginales là où elles devaient se trouver dans son modèle, c'est-à-dire dans les marges en regard du texte de Juvénal.

Après avoir présenté cet exemplaire juvénalien, il convient désormais de se confronter à l'épineuse question du modèle de ce manuscrit. L'analyse de l'ensemble de son contenu offre, en effet, quelques pistes pour cerner la bibliothèque dans laquelle les moines du Mont Saint-Michel sont allés chercher leur Juvénal autour de l'an Mil.

\section{Quelques hypothèses sur l'origine de ce matériel scolaire}

\section{Le texte de Juvénal, ses gloses et son accessus}

Comme nous l'avons vu, le texte des Satires transmis par le manuscrit se retrouve uniquement dans des témoins français, liés notamment à la région de ReimsAuxerre. Quant aux gloses et au proto-accessus qu'il renferme, ils appartiennent principalement à la branche $\varphi$ des Scholia recentior ${ }^{24}$ et au type 2 de ma

20. Cf. GrazZINI, 2011, XVIII-XIX.

21. C'est le cas, par exemple, de Schol. rec. I, 118 (1) ou II, 99 (4).

22. On trouve de telles additions, par exemple, au fol. 6r sur Sat. II, 86-87 ou au fol. 7v sur Sat. III, 9.

23. Ce «raté» s'explique peut-être par un changement de cahier dans le modèle.

24. Même si plusieurs additions (signe $D^{1}$ dans l'édition de Grazzini) ajoutent du matériel emprunté à la branche $\chi$, cf. GrazZINI, 2011, XIX. 
typologie des accessus carolingiens. Ce matériel reflète l'enseignement de Remi d'Auxerre lorsqu'il était à Auxerre ou à Reims. Il se diffuse rapidement dès le début du Xe siècle en Francie occidentale, avant de gagner l'ouest de l'Allemagne, notamment la région de Lorsch vers le milieu du $\mathrm{X}^{\mathrm{e}}$ siècle. Dans cette perspective, il est très vraisemblable que le modèle du manuscrit montois ait été copié dans le quart nord-est de la France au cours du $\mathrm{X}^{\mathrm{e}}$ siècle.

Il est intéressant de noter que certains manuscrits sont proches du manuscrit du Mont Saint-Michel à la fois pour leur texte des Satires et pour leur matériel explicatif. C'est le cas des manuscrits Leiden, UB, Voss. lat. Q 18 et Leiden, UB, Voss. lat. $\mathrm{F} 64$ ( $V$ et $B$ de l'édition Grazzini). $V$ partage, notamment, avec le manuscrit du Mont plusieurs gloses que l'on ne retrouve pas dans les autres témoins utilisés par l'édition Grazzini ${ }^{25}$. $B$, de son côté, contient un accessus proche de ceux du manuscrit du Mont. De même, le manuscrit Paris, BnF, lat. 4883A, qui ne contient pas de gloses, transmet un texte des Satires et un accessus proches de ce que l'on lit dans le manuscrit du Mont. Ce manuscrit a probablement été copié dans la région de Limoges mais une grande partie de son contenu est liée aux écoles du nord-est de la France ${ }^{26}$ et il est fort possible que son texte de Juvénal et son accessus juvénalien proviennent aussi de cette région.

Le manuscrit du Mont semble, par ailleurs, entretenir une certaine parenté avec un autre manuscrit juvénalien français encore peu étudié: le manuscrit Wien, ÖNB, 277. Ce volume, qui renferme les gloses marginales des Scholia recentiora copiées sous forme de commentaire continu, contient notamment un accessus dont plusieurs éléments rares se retrouvent aussi dans celui copié au fol. 17r-v du manuscrit du Mont (notamment la glose sur «satyra» et les premières lignes d'une Vita Iuuenalis ${ }^{27}$. Par ailleurs, certaines gloses marginales absentes des autres témoins utilisés par l'édition Grazzini se retrouvent dans le manuscrit de Vienne et dans le manuscrit du Mont. C'est le cas de cette glose portant sur Sat. I, 7 que l'on lit au fol. $17 \mathrm{v}$ du manuscrit de Paris et au fol. $2 \mathrm{v}$ du manuscrit de Vienne ${ }^{28}$ :

Sensus autem huiusmodi est: nulli magis nota est sua domus quam mihi sunt omnia nota que de Marte et Ilia et eorum progenie referuntur, necnon que de Vulcano et Iasone et Eaco iudice infernali fabulose narrantur, que per omnia plenissime scribere poteram ${ }^{29}$.

25. Par exemple, pour la première satire, Schol. rec. I, 44 (3), I, 47 (2), I, 56 (12), I, 109 (8).

26. Cf. supra, n. 12

27. Cf. Duplessis, 2017, 116 .

28. Comme le manuscrit de Vienne ne fait pas partie des témoins qu'il a utilisés, cette glose ne se trouve donc pas dans l'édition des Scholia recentiora, car Stefano Grazzini a fait le choix de n'éditer que les scholies marginales rencontrées dans au moins deux témoins et a donc laissé de côté celles présentes dans un seul manuscrit. Ce choix éditorial ne facilite pas l'étude des scholies les plus originales du manuscrit du Mont.

29. Le texte édité ici est celui du manuscrit de Paris. Voici les trois variantes du manuscrit de Vienne: sua domus] domus sua Wien; ilia] ilia romuloque Wien; per omnia] om. Wien. 


\section{Les textes annexes copiés par la main principale: Quid sit ceroma}

Quid sit ceroma a été édité pour la première fois par E. Baluze à partir du manuscrit du Mont, qui se trouvait à son époque dans la bibliothèque de Colbert $^{30}$. L'édition la plus récente est celle d'E. Dümmler dans les Monumenta Germaniae Historica, où cette pièce est placée parmi les additamenta aux lettres de Loup de Ferrières ${ }^{31}$. Ce texte est transmis par onze témoins et circule à trois reprises avec les Satires de Juvénal:

- Barcelona, Arxiu de la Corona d'Aragó, Diversos y Collecciones, Ripoll 206, fol. 159v-16or (XII; contient juste le début de la lettre);

- Cambridge, King's College, 52, fol. 61v (IX ${ }^{3 / 3}$, nord-est de la France): manuscrit de Juvénal;

- Chicago, Newberry Libr. F. 3, fol. 12v (olim Phillipps, 8462) (XI, nord de l'Italie ou sud de la France, provenance: Novalese);

- Leiden, UB, BPL, 36-II, fol. 13or (IX², lettre ajoutée au X, Lorsch);

- Leiden, UB, BPL 82, fol. 86r-v (XI, Allemagne? il manque la fin de la lettre): manuscrit de Juvénal;

- London, BL, Royal 15 B XIX-III, fol. 196v-198v (X, Reims);

- Montpellier, BIUM, H 225, fol. 56r-v (IX ${ }^{3 / 3}$, la lettre est ajoutée à la fin du IX ${ }^{e}$ siècle, France, provenance: Saint-Benoît-sur-Loire?);

- Paris, BnF, lat. 8070, fol. 124r-126r (X-XI, Mont Saint-Michel): manuscrit de Juvénal;

- Paris, BnF, lat. 12949, fol. 38v-39v (X-XI, nord de la France);

- Paris, BnF, lat. 13957, fol. 79r-v (X ${ }^{\text {in }}$, France $)^{32}$;

- St. Gallen, Stiftsbibl., 831, p. 173-175 (XI, Saint-Gall ?).

Il s'agit d'une lettre dans laquelle l'auteur explique de façon extrêmement érudite à son interlocuteur ce que désigne le mot ceroma (un onguent utilisé par les lutteurs dans l'Antiquité). Durant l'Antiquité, ce terme apparaît chez Juvénal (ceromatico, Sat. III, 68 et ceroma, Sat. VI, 246) mais aussi chez Martial, Pline l'Ancien ou Sénèque. On le retrouve chez quelques auteurs tardo-antiques, notamment dans un passage d'une lettre de Jérôme (Epist. 57), repris dans la lettre carolingienne.

Quid sit ceroma a été attribué pendant longtemps à Loup de Ferrières ${ }^{33}$, avant que Veronika von Büren propose d'y voir un travail d'Heiric d'Auxerre

30. Stephani Baluzii Miscellaneorum Liber quartus, hoc est, collectio ueterum monumentorum quae hactenus latuerant in uariis codicibus ac bibliothecis, Parisiis, 1683, p. 417-420.

31. Lupus Ferrariensis, Epistolae. Additamentum, éd. E. Dümmler, Berlin (MGH; Epp. 6), 1925, p. 114-117.

32. La présence de cette lettre dans ce manuscrit a été signalée récemment par Luca Paretti, cf. Paretti, 2013.

33. BEESON, 1938. 
(notamment parce que, selon elle, le plus ancien témoin du texte transmet aussi les gloses d'Heiric sur Juvénal) ${ }^{34}$. Récemment, Luca Paretti, qui ne semble pas connaître l'hypothèse de V. von Büren, a proposé de l'attribuer à Sédulius Scottus et considère que c'est l'occurrence hiéronymienne qui serait à l'origine de la rédaction de cette lettre érudite ${ }^{35}$. En attendant une nouvelle édition du texte ${ }^{36}$, qui permettrait peut-être de préciser l'identité de l'auteur de ce texte et l'origine du modèle de l'exemplaire du Mont Saint-Michel, je me contenterai ici de quelques remarques permettant de situer quelque peu la place de ce témoin dans la tradition de cette lettre ${ }^{37}$.

Tout d'abord, pour clarifier l'histoire de la transmission de ce texte, il est possible de rapprocher certains témoins manuscrits entre eux. Paris, BnF, lat. 13957 et St. Gallen, Stiftsbibl., 831 présentent des variantes similaires et Quid sit ceroma y est copié à côté d'une lettre sur le comput qu'on ne trouve que dans ces deux témoins ${ }^{38}$. De même, le manuscrit de Barcelone et celui de Chicago semblent appartenir à la même branche car ils transmettent tous deux Quid sit ceroma avec la lettre attribuée à Remi d'Auxerre sur les Hongrois ${ }^{39}$. Il s'agit des deux seuls témoins à ne pas transmettre cette lettre avec deux autres lettres attribuées à Remi. Enfin, il convient aussi de rapprocher les deux manuscrits de Juvénal Cambridge, King's College 52 et Leiden, UB, BPL 82, dans lesquels la lettre érudite a été copiée juste après la fin des Satires. Veronika von Büren a signalé que les gloses du manuscrit de Leiden étaient une copie directe de celles du manuscrit de Cambridge ${ }^{40}$. Même si le texte de Quid sit ceroma transmis par le manuscrit de Leiden présente plusieurs petites variantes par rapport à celui du manuscrit de Cambridge, il semble s'agir à chaque fois de simples erreurs de copie. On peut donc supposer que le manuscrit de Cambridge a aussi servi de modèle au manuscrit Leiden, UB, BPL 82 pour Quid sit ceroma.

Cette association des Satires à Quid sit ceroma se retrouve dans un seul autre manuscrit, l'exemplaire copié au Mont Saint-Michel, qui copie lui aussi la lettre sur «ceroma» juste après l'explicit des Satires. Cette caractéristique nous invite à rapprocher le modèle du manuscrit normand des écoles du nord-est

34. BÜREN, 2010a, p. 390-391. Ludwig Traube avait déjà prudemment avancé l'hypothèse que l'auteur de cette lettre devait être un membre de l'école de Loup de Ferrières camarade d'Heiric d'Auxerre: Traube, 1893, p. 93, 99-100 et 724-725.

35. Quid sit ceroma fait, cependant, très certainement référence à Juvénal (Sat. II, 52) en évoquant les colyphia mangés par les athlètes (éd. Dümmler, p. 116, 1. 20-21). L. Traube, pour sa part, a dans un premier temps considéré que la rédaction de cette lettre était liée au proverbe cité par Jérôme avant de se raviser en soulignant les liens probables avec l'exégèse de Juvénal (cf. TRAUBE, 1893, p. 99-100 et les additions faites à la p. 725).

36. L'édition de Dümmler n'utilise ni le manuscrit de Montpellier, un des plus anciens témoins du texte, ni le manuscrit Leiden, UB, BPL 82, ni celui de Barcelone, ni celui signalé par L. Paretti (Paris, BnF, lat. 13957).

37. Je développe davantage certains points concernant l'histoire de ce texte dans DupLEssis, 2020, p. 203-206.

38. Sur la proximité de ces deux témoins, je renvoie à l'étude de L. Paretti.

39. Voir JEUdy, 1991, p. 497-499.

40. Cf. BÜREN, 2O10b. 
de la France d'où provient l'exemplaire de Cambridge, même si les gloses juvénaliennes contenues dans le volume du King's College n'appartiennent pas aux Scholia recentiora de Remi d'Auxerre mais correspondent à une strate exégétique antérieure que V. von Büren propose d'attribuer à Heiric d'Auxerre, mais qui pourrait très bien être une strate proto-rémigienne, comme le propose Stefano Grazzini dans l'introduction de son édition à paraittre de ces gloses.

Par ailleurs, on peut noter que Paris, BnF, lat. 8070 ne donne pas de titre à cette lettre. Il partage cette caractéristique avec six autres manuscrits, parmi lesquels on trouve les plus anciens témoins du texte: Paris, BnF, lat. 13957 et St. Gallen, Stiftsbibl., 831, Cambridge, King's College, 52 et Leiden, UB, BPL 82, ainsi que Montpellier, BIUM, H 225 et Leiden, UB, BPL, 36.

Si l'on se penche sur les variantes textuelles, on constate qu'elles sont rarement conséquentes. On peut, néanmoins, en signaler trois intéressantes dans le manuscrit du Mont ${ }^{41}$ :

- comme dans cinq autres témoins ${ }^{42}$, on trouve copié en toutes lettres le nom de l'intermédiaire Fredilo (éd. Dümmler, p. 115, 1. 12). Le manuscrit de Montpellier en conserve simplement les deux premières lettres, tandis que le manuscrit Leiden, UB, BPL 36 l'omet complètement, tout comme le manuscrit de Londres, qui, à la place de noster Fredilo, contient abréviation $n r t$ avec un tilde au-dessus du " $\mathrm{t}$ " (plus rare que l'abréviation $n \tilde{r}, n r \tilde{t}$ se rencontre parfois à l'époque carolingienne, notamment dans le nord-est de la France $)^{43}$. Dans le manuscrit de Saint-Gall, on lit noster Fredili (avec la haste du $d$ barrée), tandis que dans Paris, BnF, lat. 13957, malgré une perte matérielle, on parvient à distinguer noster Fredil ou noster Fredili (avec la haste du $l$ barrée);

- comme dans les manuscrits de Londres et de Chicago et dans Leiden, UB, $\mathrm{BPL} 36$, on trouve reiciuntur (éd. Dümmler, p. 116, 1. 19), là où la moitié des manuscrits a redduntur ou referuntur. On lit, en effet, redduntur dans quatre manuscrits: Cambridge, King's College, 52, Paris, BnF, lat. 12949, Paris, BnF, lat. 13957 et Saint-Gall, 831 ${ }^{44}$. Quant à referuntur, cette variante se lit dans le manuscrit de Montpellier, qui compte de nombreuses leçons originales;

- eligeritis au lieu de digessi (éd. Dümmler, p. 117, 1. 10). Cette leçon le rapproche peut-être du manuscrit de Londres et du Leiden, BPL, 36 qui ont diligessi ${ }^{45}$, ainsi que du manuscrit de Montpellier qui a la leçon diligo.

41. L'intérêt des deux dernières variantes était déjà mis en avant dans OrLANDI, 2008, p. 444-445.

42. Barcelona, Arxiu de la Corona d'Aragó, Diversos y Collecciones, Ripoll 206, Cambridge, King's College, 52, Chicago, Newberry Libr. F. 3, Leiden, UB, BPL 82, Paris, BnF, lat. 12949.

43. L'apparat de Dümmler indique de façon erronée que ce manuscrit contient juste la première lettre « $\mathrm{F} »$

44. Cette leçon se trouvait sans doute aussi dans le manuscrit Leiden, UB, BPL 82, qui semble descendre du manuscrit de Cambridge, mais la perte de la fin de la lettre dans cet exemplaire nous empêche de nous en assurer.

45. Contrairement à ce qu'indique l'apparat de Dümmler, le manuscrit de Saint-Gall n'a pas diligessi mais bien digessi. 
Ces variantes textuelles ne permettent pas de rapprocher le texte du manuscrit du Mont d'un autre témoin en particulier mais nous invitent à conclure au moins qu'il n'appartient pas à la même branche que le manuscrit de Saint-Gall et Paris, BnF, lat. 13957.

Quoi qu'il en soit, la diffusion de Quid sit ceroma semble partir du nord-est de la France, puis toucher la région de Saint-Gall, le nord de l'Italie, la Catalogne et l'ouest de l'Allemagne. Ce réseau de diffusion est commun à plusieurs autres textes scolaires liés aux écoles du nord-est de la France comme les Scholia recentiora sur Juvénal ${ }^{46}$, les Scholica Graecarum Glossarum ${ }^{47}$ ou l'Epitaphium Diogenis $^{48}$. Là encore, il semble probable que ce soit de cette première zone de diffusion que provienne le modèle du manuscrit du Mont.

\section{Les textes annexes copiés par la main principale:} la Genealogia Enee et Priami

Juste après Quid sit ceroma, on trouve dans Paris, BnF, lat. 8070 un autre texte scolaire transcrit par la même main, que je propose de nommer Genealogia Enee et Priami: il s'agit d'une généalogie d'Énée, copiée sans titre aux fol. 126r-127r. Cette pièce érudite permettait d'expliquer d'une part pourquoi Énée, sans être fils de Priam, appartient à la famille royale troyenne, d'autre part pourquoi Énée affirme que les Troyens et les Grecs ont le même ancêtre (Aen. VIII, 127-142) et enfin pour quelle raison Junon s'acharne par jalousie contre les Troyens, qu'elle qualifie de genus inuisum en Aen. I, 28 (notamment parce que leur ancêtre Dardanus est le fruit des amours adultères de Jupiter et d'Electra).

Les phrases 6 à 15 de la Genealogia Enee et Priami, où sont exposés les descendants de Dardanus, sont fortement apparentées à l'Origo Troianorum, une généalogie que l'on trouve dans plusieurs manuscrits de Virgile ${ }^{49}$, et à un chapitre du Premier Mythographe du Vatican ${ }^{50}$, qui reprend probablement l'Origo Troianorum. Ce traité mythographique n'est conservé que dans un seul manuscrit copié au XII siècle probablement en France (Vaticano, BAV, Reg. lat. 1401). L'Origo Troianorum est conservée dans de nombreux témoins, dont voici les plus anciens:

- Hamburg, Staats-und Universitätsbibliothek, Cod. in Scr. 52, fol. 54 r (IX ${ }^{2 / 4}$, Paris? lié peut-être à un exemplaire de Saint-Médard de Soissons) ${ }^{51}$;

46. Cf. Grazzini, 2011, p. XV-Xxxix.

47. Cf. Lendinara, 2011.

48. Cf. Duplessis, 2019.

49. Cf. Munk Olsen, 1985, p. 689 (\#207 et \#211) et Ottaviano, 2013-2014, p. 303-304 (avec édition du texte).

50. Mythographus Vaticanus I, éd. P. Kulcsár, Turnhout, Brepols, 1987 (CCSL; 91C), cap. 132, p. 55, 1. 5-16 = Le premier mythographe du Vatican, II, cap. 33 (3-7), éd. N. Zorzetti, trad. J. Berlioz, Paris, Les Belles Lettres (CUF), 1995, p. 77. La datation de ce texte fait encore débat. La source la plus récente qu'il utilise est le commentaire de Remi d'Auxerre sur la Consolation de Philosophie de Boèce rédigé au plus tard dans les premières années du $\mathrm{X}^{\mathrm{e}}$ siècle.

51. Sur ce manuscrit, voir Munk Olsen, 1985, p. 722 et Ottaviano, 2013-2014, p. 219-221. Pour le lien possible avec Saint-Médard, voir SAVAGE, 1934, p. 180. 
- Laon, Bibl. mun., 468, fol. 4r-v (IX ${ }^{3 / 4}$, Laon $)^{52}$;

- Leiden, UB, Voss. lat. F 25, fol. 44r (X'1, France $)^{53}$;

- Paris, BnF, lat. 7926, fol. 1r (IX ${ }^{2 / 4}$, Fleury ou plus vraisemblablement SaintGermain d'Auxerre; l'Origo est ajoutée à la fin du $\mathrm{IX}^{\mathrm{e}}$ ou au début du $\mathrm{X}^{\mathrm{e}}$ siècle $)^{54}$;

- Paris, BnF, lat. 10307, fol. 96r (IX ${ }^{4 / 4}$, nord-est de la France, probablement Laon $)^{55}$;

- Wolfenbüttel, HAB, Gud. lat. 70, fol. 54v (IX ${ }^{2 / 4}$, Lyon ou Allemagne; l'Origo est une addition de la seconde moitié du IX ${ }^{\mathrm{e}}$ siècle $)^{56}$.

Ces trois textes exposent de la même façon les généalogies de Priam et d’Énée, alors qu'il existait plusieurs variantes des lignées troyennes ${ }^{57}$. Mais le début et la fin de la généalogie du manuscrit du Mont ne se retrouvent ni dans l'Origo ni dans le Premier Mythographe. La Genealogia Enee et Priami montoise se révèle, en effet, être extrêmement rare. Elle n'était connue, sauf erreur de ma part, que dans un exemplaire de Virgile conservé aujourd'hui à Montpellier: Montpellier, BIUM, $\mathrm{H} 253$ (IX ${ }^{2 / 3}$, nord-est de la France), où la généalogie des Troyens aurait été ajoutée au fol. 12or à la fin du IX ${ }^{\mathrm{e}}$ siècle d'après Bernhard Bischoff ${ }^{58}$. J'ai retrouvé, par ailleurs, un troisième témoin de cette Genealogia Enee et Priami dans un recueil historique copié vers 1200 dans l'abbaye de Jumièges et aujourd'hui conservé à Rouen (Rouen, Bibl. mun., 1177 [U.74], fol. 289r-v), où la généalogie des Troyens est insérée au début de l'Historia Brittonum de Nennius au sein de la généalogie du peuple breton ${ }^{59}$. On trouvera en annexe une édition de ce texte à partir de ces trois témoins complets et des témoins partiels que sont le Premier Mythographe du Vatican et l'Origo Troianorum.

Il est difficile de déterminer avec certitude les relations entre les trois témoins de la Genealogia Enee et Priami. Le manuscrit de Montpellier a plusieurs leçons qui le séparent des deux autres, notamment sa phrase introductive, ce qui nous invite

52. Ce manuscrit est le seul témoin de l'Origo à ne pas être un manuscrit de Virgile, il s'agit d'un grand recueil scolaire utilisé par les maîtres de l'école de Laon, cf. ConTrenI, 1984.

53. Munk Olsen, 1985, p. 727 et Bischoff, 2004, p. 50.

54. Sur ce manuscrit, voir Munk Olsen, 1985, p. 754-755 et Ottaviano, 2013-2014, p. 233-244. La provenance auxerroise semble confirmée par plusieurs annotations signalées dans la notice de Franck Cinato sur Archives et manuscrits (https://archivesetmanuscrits.bnf.fr/ark:/12148/ cc11298h; page consultée le 15 janvier 2019).

55. Sur ce manuscrit, voir Munk Olsen, 1985, p. 764-765 et Ottaviano, 2013-2014, p. 270-276.

56. La généalogie des Troyens a été copiée en marge d'Aen. VII, p. 207-208, où est raconté le voyage de Dardanus. Sur ce manuscrit, voir Bischoff, 2004, p. 501-502 et Ottaviano, 2013-2014, p. 288-291. La présence de l'Origo dans ce manuscrit n'avait pas été notée par S. Ottaviano, qui ne prend donc pas en compte ce témoin dans son édition de ce texte (ibid., p. 304).

57. Voir, par exemple, Servius (auctus), Aen. VIII, 130, éd. Thilo-Hagen, p. 219, 1. 3-6; Servius (auctus), Georg. III, 35 éd. Thilo, p. 278, 1. 1-4; Dictys, Ephemeris, IV, 22, éd. Eisenhut, p. 100, 1. 4-12.

58. Cf. Bischoff, 2004, p. 205.

59. On trouve le même type de généalogie bretonne augmentée avec la matière troyenne dans d'autres exemplaires de l'Historia Brittonum: par exemple, Cambridge, University Library, Ff.1.27, p. 22 (XII-XIII, Angleterre) ou Cambridge, Corpus Christi, 363, fol. 1r (XIV ou XV). 
à penser qu'il ne doit pas être le modèle du manuscrit montois. Les manuscrits de Rouen et de Paris ont plusieurs leçons en commun qui les séparent du manuscrit de Montpellier ${ }^{60}$. En revanche, les leçons qui rapprochent le manuscrit de Rouen de celui de Montpellier sont minimes ${ }^{61}$. Il est donc clair que le texte de Rouen est plus proche de celui du Mont que de celui de Montpellier mais il est néanmoins délicat d'affirmer que le manuscrit de Rouen descend avec certitude de celui de Paris. Le manuscrit de Rouen a, en effet, plusieurs leçons qui lui sont propres. Si on laisse de côté les deux additions qui visent à rattacher la figure de Brutus à la lignée d'Énée afin de transformer la généalogie des Troyens en généalogie des Bretons $^{62}$, il subsiste quelques différences conséquentes entre le texte du manuscrit de Paris et celui de Rouen:

(2) iuppiter Montep. Paris. $\neq$ iupiter saturni filius celii filius Rotom.

(3) iouis Montep. Paris. $\neq$ iupiter Rotom.

(9) troiam apellari Montep. Paris. Orig. $\neq$ urbem troiam suo nomine appellari Rotom.

(16) reuertitur Montep. Paris. $\neq$ reuoluitur Rotom.

À ces quatre principales divergences, il convient d'ajouter les variantes minimes qui sont relativement nombreuses. Il s'agit principalement de légères reformulations ${ }^{63}$, d'inversions dans l'ordre des mots $^{64}$, de petites additions ${ }^{65}$ ou omissions ${ }^{66}$. Ces différences peuvent s'expliquer par l'interventionnisme du copiste de Jumièges ou par l'existence d'une copie intermédiaire (aujourd'hui perdue). Mais leur nombre nous invite à envisager aussi que le manuscrit de Rouen ne soit pas un descendant direct de la copie montoise de la Genealogia Enee et Priami.

Pour poursuivre l'étude des liens entre ces deux copies normandes, il faudrait étendre notre enquête à l'ensemble des textes contenus dans Rouen, Bibl. mun., 1177, dont voici une description synthétique ${ }^{67}$ :

60. (1) sic ordinatur genealogia eneae et priami qui expugnatus est in troia Paris. Rotom. $\neq$ quomodo coniungitur genealogia aeneae et priami qui expugnatus est in troia sic ordinatur Montep. (2) iuppiter Paris. Rotom. $\neq$ iouis Montep. (6) dilatus est Paris. Rotom. $\neq$ delatus Montep. (11) ili Paris. Rotom. $\neq$ ilo Montep. (14) capis anchisen Paris. Rotom. $\neq$ ex quo natus est anchises Montep. (version proche de Orig. et de Myth.).

61. (10) habuit filios] filios habuit Montep. Rotom. Myth. (12) expugnatus] est add. Montep. Rotom.

62. (15) eneas aschanium aschanius siluium siluius brutum a quo britones dicuntur et originem ducunt post procreauit add. Rotom. (17) eneas filius anchise] brutus filius siluii filii aschanii filii enee filii anchise Rotom.

63. (2) id est maiam] maiam scilicet Rotom. (5) et - amor] fuit autem apud iouem amor maior Rotom. (6) uero] namque Rotom.

64. (2) habuit duas] duas habuit Montep. | filias athlantis] athlantis filias Rotom. (4) est - omnium] omnium grecorum est origo Rotom. (8) hisdem - regnauit] isdem regnauit locis Rotom. (9) sui nominis] nominis sui Rotom.

65. (5) fuit post troianorum add. Rotom. (9) iustitia] in iusticia Rotom. (11) laomedon] hic laomedon Rotom.

66. (3) et] om. Rotom. (10) autem] om. Rotom. (15) autem] om. Rotom. L'omission de la phrase $\mathrm{n}^{\circ} 7$ s'explique par un saut du même au même.

67. Sur ce manuscrit, je renvoie à l'importante contribution de David N. Dumville (Dumville, 1985). 
- fol. 1-59ra: Geoffroy de Monmouth, Historia Regum Britanniae;

- fol. 59ra-62ra: Extraits de l'Historia Ecclesiastica de Bède;

- fol. 62ra-166r: Historia Anglorum libri decem d'Henry de Huntingdon;

- fol. 166v-171r: Extraits de la chronique de Robert de Torigni (pour les années 1147-1157);

- fol. 171r-172v: Continuation de la chronique de Robert pour les années 1157$1160^{68}$

- fol. 173r-275r: Bède, Historia ecclesiastica Gentis Anglorum;

- fol. 276r-278r: deux fragments du livre X de l'Historia Anglorum d'Henry de Huntingdon;

- fol. 282v-288v: Bède, De natura rerum;

- fol. 278v-282v, 299r-302r: Bède, De temporibus liber;

- fol. 289r-297v: Historia Brittonum de Nennius dans la version du pseudoGildas.

Dans ce grand recueil, qui renferme principalement des textes historiques sur l'Angleterre, deux particularités retiennent l'attention, la présence du $D e$ natura rerum de Bède et le titre donné à l'Historia de Nennius dans ce volume: "Incipit liber Gilde sapientis de primis habitatoribus Britannie, que nunc dicitur Anglia, et de excidio ejus» (fol. 289r). Ces deux caractéristiques le rapprochent d'un manuscrit du Bec aujourd'hui perdu que l'on connaît, notamment, grâce au catalogue du XII ${ }^{e}$ siècle et au catalogue mauriste de $1693^{69}$ :

- Catalogue médiéval, item $80^{70}$ : In alio historia Anglorum libri V, De temporibus liber I minor, De Naturis Rerum liber I, Liber Gilde sapientis de excidio Britannie, Vita sancti Neoti, que in capite ponitur;

- Catalogue de 1693, item 137 (Paris, BnF, lat. 13068, fol. 52r) : Bedae Venerabilis de historia Anglorum lib. 5. in- $4^{\circ}$. Idem de temporibus, horis et momentis minor. Ejusdem libellus de natura rerum, seu de quadrifario opere Dei ex libro Plinii secundi naturae historiae. Prisciani versus de signis caelestibus et de quatuor ventis. Beda Venerabilis de conversione et conversatione sua; necnon de numero opusculorum suorum ex lib. 5. hist. Angl. Gildae sapientis liber de primis habitatoribus Britanniae, quae nunc dicitur Anglia et de excidio ejusdem.

On retrouve dans ce volume du Bec plusieurs ouvrages de Bède contenus dans le manuscrit de Rouen et le pseudo-Gildas avec le même titre. L'association de ces différentes œuvres de Bède avec l'Historia de Nennius est très rare, le titre donné à cet ouvrage l'est encore plus. Il n'est attesté que dans ces deux

68. Cette continuation a été éditée par Léopold Delisle (DelisLe, 1873, 165-170). On la retrouve dans d'autres manuscrits normands (ibid., 143-146).

69. Il existe un autre catalogue moderne du Bec, mais la description qu'il donne de ce volume est moins développée (Paris, BnF, lat. 11777, fol. 287r, item f.72).

70. Ce catalogue a été récemment réédité par Laura Cleaver (CLEAvER, 2018, p. 195-205). 
exemplaires. David N. Dumville signale, néanmoins, que l'on trouve un titre apparenté («Incipiunt excerptiones de Libro Gilde Sapientis quem composuit de primis habitatoribus Brittanie que nunc Anglia dicitur et de excidio eius») dans trois témoins normands appartenant à la même sous-branche du texte de l'Historia Brittonum ${ }^{71}$ :

- Leiden, UB, BPL $20\left(\mathrm{XII}^{2 / 4}\right.$, Le Bec);

- London, BL, Cotton Nero D VIII (XII², Angleterre, Colchester?);

- Évreux, Bibl. mun., 41 (XIII, Lyre).

D'après David N. Dumville, le titre de ces trois témoins correspondrait à une version légèrement révisée du texte tel qu'on peut le lire dans l'actuel manuscrit de Rouen et qu'on pouvait le lire dans le manuscrit perdu du Bec. Rien ne permet d'affirmer que l'interpolation troyenne se trouvait dans cet exemplaire perdu (elle est absente de la généalogie des Bretons dans les manuscrits Leiden UB, BPL 20, fol. 101v, London, BL, Cotton Nero D VIII, fol. 63v et Évreux, Bibl. mun., $41,138 \mathrm{v})$, mais, à titre d'hypothèse, on peut imaginer que ce volume du Bec contenait peut-être aussi la Genealogia Enee et Priami et qu'il pourrait s'agir d'un intermédiaire entre la copie du Paris, BnF, lat. 8070 et la copie du manuscrit de Rouen. L'existence d'un intermédiaire lié au Bec pourrait expliquer plusieurs des principales divergences textuelles entre ces deux témoins normands. Il est possible que ce petit texte sur les ancêtres d'Énée ait suscité l'intérêt d'un intellectuel normand au cours du XII ${ }^{\mathrm{e}}$ siècle et que sa diffusion soit liée aux échanges intellectuels entre le Mont, le Bec et Jumièges à cette époque ${ }^{72}$.

Quoi qu'il en soit des relations entre le manuscrit de Rouen et celui de Paris, le témoin le plus ancien de la Genealogia Enee et Priami nous renvoie une fois de plus vers les écoles du nord-est de la France, d'où proviennent la plupart des plus anciens témoins de l'Origo Troianorum (liés aux écoles de Laon, Soissons et Auxerre). Un autre indice rapproche la généalogie copiée dans le manuscrit du Mont des écoles du nord-est de la France. Il s'agit d'une glose marginale sur Aen. I, 28, copiée au fol. 56v du manuscrit Valenciennes, Bibl. mun., 407 (IX², Saint-Amand ${ }^{73}$ :

Genus inuisum id est genus Troianum, quia ex pelice Iunonis, id est Electra concubina Iouis, natus est Dardanus, unde orti sunt Troiani. Concubuit namque Iuppiter cum Electra filia Atlantis et genuit Dardanum. Dardanus Erictonium, Erictonius Troem, Trous Ilum, Assaracum et Ganimedem. Ganimedes non apparuit quia tulit eu<m> deus, id est Iuppiter. Assaracus [Ilus a.c.] genuit Capin, Capis Anchisen, Anchises Aenean ex Venere, Assaracus ${ }^{74}$ genuit Laomedontem, Laomedon genuit Priamum,

71. DumVille, 1985, p. 5-6.

72. Le contenu des manuscrits nous inviterait, notamment, à rapprocher ce dossier de l'activité de Robert de Torigni.

73. Je remercie Michael Allen de m'avoir aidé à résoudre les notes tironiennes utilisées dans cette glose.

74. Ilus et d'Assaracus semblent avoir été inversés mais seul le premier nom aura été corrigé. Il faudrait ici remplacer Assaracus par Ilus. On retrouve la même généalogie avec la même inversion dans une glose du fol. 156r (cf. note suivante). 
Priamus genuit Paridem et Hectorem, ex quibus omnes Troiani; idem Iuppiter concubuit cum Maia sorore Electrae et genuit Mercurium, ex quo omnes Greci.

On retrouve ici la même généalogie des Troyens et la même insistance sur la figure de Mercure qui serait «l'ancêtre de tous les Grecs» (idée absente de l'Origo et du Premier Mythographe $)^{75}$. Cela semble confirmer le lien entre le contenu du manuscrit Paris, BnF, lat. 8070 et les écoles de la province ecclésiastique de Reims.

\section{Les ajouts des fol. $127 v-128 v$}

Les mains $\mathrm{C}$ et $\mathrm{D}$ ont ajouté différents extraits et petites notes de travail sans rapport direct avec l'étude de Juvénal et des classiques. Les additions notées par la main $\mathrm{C}$ aux fol. $127 \mathrm{~V}-128 \mathrm{r}$ forment un petit florilège sur la logique et la division des savoirs. Même si l'analyse des extraits ajoutés par $\mathrm{C}$ ne permet pas réellement d'éclairer l'origine du matériel copié par A et B - il est difficile de savoir si ces notes ont été prises à partir de manuscrits montois ou sur des manuscrits provenant d'autres bibliothèques -, il m'a paru intéressant de transcrire ce florilège inédit, car son contenu, davantage lié à l'enseignement de la dialectique et de la philosophie qu'à l'étude des classiques et de la grammaire, illustre d'autres aspects de la vie intellectuelle montoise vers l'an Mil. Voici donc une transcription des différents éléments de cette compilation (pour les trois premiers extraits d'Augustin, j'indique entre crochets les variantes du manuscrit du Mont par rapport au texte de l'édition Mutzenbecher dans le Corpus Christianorum):

$[127 \mathrm{v}]$

$<1>$ VTRVM CORPVS A DEO SIT. Augustinus in libro de diuersis questionibus: omne bonum a deo, omne speciosum bonum, in quantum speciosum est, et omne quod species continet speciosum est. Omne autem corpus, ut corpus sit, specie aliqua continetur. Omne igitur corpus a deo.

[Augustin, De diuersis quaestionibus octoginta tribus, 10] ${ }^{76}$

$<2>$ VTRVM ANIMA A SE IPSA SIT. Omne uerum a ueritate uerum est; et omnis anima eo anima est, quo uera [et add. cod.] anima est. Omnis igitur anima a ueritate habet, ut omnino anima sit. Aliud autem anima est aliud ueritas; nam ueritas falsitatem numquam patitur, anima uero sepe fallitur. Non igitur, cum a ueritate anima est, a se ipsa est. Est autem ueritas deus. deum igitur habet auctorem ut sit anima.

[Augustin, De diuersis quaestionibus octoginta tribus, 1]

75. Cette mise en avant de l'origine commune des Grecs et des Troyens s'inspire du discours d'Énée à Évandre (Aen. VIII, 127-142). On retrouve d'ailleurs dans le manuscrit de Valenciennes une généalogie très proche de cette glose en marge de la rencontre d'Énée avec Évandre (fol. 156r) et certaines des formulations données par le début de la Genealogia peuvent aussi provenir de commentaires non serviens sur ce passage. Par exemple, Donat, Interp. Virg., Aen. 8, éd. Georgii, p. 136, 1. 2-6: «Electra genuit Dardanum et Maia Mercurium, Dardanus dedit Troianis originem et Mercurius Arcadibus, perinde unus auus est ambobus, Dardano scilicet et Mercurio fratribus consobrinis".

76. Cette œuvre est relativement répandue à cette époque. Nous avons conservé une copie montoise du XII ${ }^{e}$ siècle (Avranches, Bibl. mun., 83, fol. 232-276). 
$<3>$ De InTELLECTV. Omne quod se intellegit conprehendit se; quod autem se conprehendit finitum est sibi; et intellectus [intellectus om. cod.] intellegit se, [et intellectus add. cod.] ergo finitus est sibi. Nec infinitus esse uult, quamuis possit, quia notus sibi esse [esse sibi cod.] uult; amat enim se.

[Augustin, De diuersis quaestionibus octoginta tribus, 15]

$<4>$ DE SEPTIFORMI GRATIA. In mente enim nostra primus ascensionis gradus est timor Domini, secundus pietas, tertius scientia, quartus fortitudo, quintus consilium, sextus intellectus, septimus sapientia.

[Grégoire le Grand, Homiliae in Hiezechihelem prophetam, II, 7, éd. Adriaen, p. 321, 1. 216-219] ${ }^{77}$

$<5>$ Augustinus: Neque enim nullum peccatum est ea quae lex dei prohibet concupiscere atque ab his abstinere timore poene non amore iusticiae. 'Neque nullum peccatum est' id est magnum peccatum est. Si enim dixisset 'neque ullum', tunc debuisset intelligi 'nullum'. Nunc uero duo negatiua, id est 'neque nullum', unum faciunt adfirmatiuum.

[citation d'Augustin, De ciuitate Dei, 14, 10 (éd. Dombart-Kalb, p. 430, 1. 21-23), suivie d'une explication sur le sens de la double négation dans ce passage $]^{78}$

$<6>$ Ypotetici syllogismi fiunt cum adiectione coniunctionis [correxi, coniunctiones cod.] qui sunt septem. Primus modus est: 'Si dies est, lucet. Est [correxi, si cod.] autem dies, lucet igitur'. Secundus modus est: 'Si dies est, lucet. Non lucet, non est igitur dies'. Tercius modus est: 'Non et dies est et non lucet. Atqui dies est, lucet igitur'. Quartus modus est: 'Aut dies est aut [aut om. cod.] nox. Atqui [correxi, autque cod.] est dies, non igitur nox [correxi, rex cod.] est'. Quintus modus est: 'Aut dies est aut nox, atqui [correxi, aut quia cod.] nox non est, dies igitur est'. Sextus modus est: 'Non et dies est et non lucet. Dies autem est, nox igitur non est'. Septimus modus est: 'Non et dies est et nox. Atqui nox non est, dies igitur est'.

[présentation des syllogismes hypothétiques tirée soit d'Isidore, Etym. II, 28, 23-25 soit de Cassiodore, Instit. II, 3, 13, éd. Mynors, p. 118, 1. 26-p. 119, 1. 10] $]^{79}$

$<7>$ Philosophia est diuinarum humanarumque, quantum homini possibile est, probabilis sciencia. Aliter philosophia est ars artium et disciplina disciplinarum. Item philosophia est meditacio mortis. Inspectiua dicitur qua supergressi uisibilia de diuinis aliquid et caelestibus contemplamur.

[définitions de la philosophie tirées soit d'Isidore, Etym. II, 24, 9 et 11 soit de Cassiodore, Instit. II, 3, 5-6, éd. Mynors, p. 110, 1. 15-18 et p. 111, 1. 3-4]

$<8>$ Planum, id est lucidum et apertum. [glose sur planum]

77. Cet extrait de Grégoire est repris par Ildefonse de Tolède (De cognitione baptismi, 130), Beatus de Liébana (Commentarius in Apocalypsin, XII, 2, 66), Raban Maur (Commentaria in Ezechielem, 15, 40) et Amalaire de Metz (De ecclesiasticis officiis, I, 27).

78. Je n'ai pas trouvé de source pour cette explication scolaire, il s'agit peut-être d'une glose marginale copiée dans un exemplaire de La cité de Dieu. Dans son Periphyseon, Jean Scot reprend l'ensemble du passage de La cité de Dieu où apparaît cette phrase.

79. On trouve un développement proche dans le prologus ad Paterium (de hypotheticis syllogismis) du commentaire de Boèce sur les Topica de Cicéron (prologue du livre V). 
On constate que ces différentes entrées reflètent un travail d'excerption (les trois premières proviennent du même texte ${ }^{80}$, comme les $n^{\circ} 6$ et 7 ) et correspondent parfois à de simples gloses sans doute glanées dans la marge ou l'interligne d'un manuscrit. Il est possible d'établir plusieurs rapprochements entre ces différents extraits. Par exemple, les trois premiers extraits tirés d'Augustin ont la forme d'un syllogisme, figure qui est au cœur de l'extrait $\mathrm{n}^{\circ} 6$. Le terme intellectus se trouve dans les extraits 3 et 4 . Les extraits 4 et 6 révèlent un intérêt pour les listes, que l'on trouvait déjà dans une glose marginale du fol. 15v (copiée par la main B):

Quot sunt claues quibus aperitur homini<s> sapientia? IIII. Prima est industria legendi. Sedulitas interrogandi. Contemptio facultatum mundi. Honor doctoris.

Ce genre d'énoncé, typique de la pédagogie médiévale, peut avoir été extrait à l'origine d'un traité construit sur l'alternance questions/réponses et circule fréquemment avec d'autres listes. On trouve plusieurs versions de ce genre d'énumération où le nombre de «clefs de la sagesse» varie entre trois et cinq. J'en donnerai simplement deux exemples:

- Vaticano, BAV, Reg. lat. 1560, fol. 137v (X ${ }^{\text {in }}$, France: commentaire sur les Disticha Catonis): Tria sunt claues sapientiae: una est dilectio dei, secunda studium discipuli, tertia honor et timor magistri;

- Titulus quare dicitur, éd. Jeudy, p. 67, 1. 27-29 ${ }^{81}$ : Quot sunt claues sapientiae? .V. Quae? Assiduitas legendi, memoria retinendi, contemptus diuitiarum, honor magistri, cotidiana interrogatio.

La version que l'on trouve dans le manuscrit du Mont dénombrant quatre clefs est plus rare. Elle semble remonter à une source d'origine insulaire ${ }^{82}$. On trouve des listes proches dans le Collectaneum miscellaneum de Sedulius Scottus, dans un florilège de Freising du VIII siècle et dans une collection hiberno-latine de la seconde moitié du VIII ${ }^{e}$ siècle:

Sedulius Scottus, Collectaneum miscellaneum, 2, 7, éd. Simpson, p. 11, 1. 33-34: Quatuor claues sunt sapientiae: industria legendi, asiduitas interrogandi, contemptus pecuniarum, honorificatio doctorum.

Florilegium Frisingense, 439, éd. Lehner, p. 36: Industria legendi et adsiduitas interrogandi et contemptus diuitiarum et honorificatio doctorum quattuor claues sunt sapientiae.

80. Il est intéressant de constater que les trois mêmes extraits du De diuersis quaestionibus octoginta tribus ont aussi été sélectionnés à la suite par Gottschalk d'Orbais dans ses notes de travail sur la nature de l'âme (Quaestiones de anima, p. 287, éd. Lambot). Ces notes sont conservées dans le manuscrit Bern, BB, 584, fol. 110v-111r (IX ${ }^{4 / 4}$, Reims). Sur le contenu des notes de Gottschalk conservées dans ce manuscrit, voir Pezé, 2017.

81. Le début de ce texte est édité dans Jeudy, 1978

82. Cf. Sims-Williams, 199o, p. 335-336; LaW, 1995, p. 126-127 (n. 3); Virgilius Maro grammaticus, Opera omnia, éd. B. Löfstedt, Leipzig, K. G. Saur (Bibliotheca Teubneriana), 2003, series 1, fragm. 216, p. 246, 1. 15 . 
Pseudo-Isidore, Liber de Numeris (München, BSB, Clm 14392, 95r): Quattuor modis claues sapientiae haec sunt: sedulitas legendi, adsiduitas interrogandi, honor magistri, contemptio mundi.

Au fol. $128 \mathrm{v}$, une quatrième main (la main D) a copié deux séries de differentiae entre lesquelles est inséré un petit glossaire. Voici la transcription de cette compilation lexicographique:

$<1>$ Inter gramma et grammaticam et grammetum et grammatum et grammaticum quid interest? Gramma greco sermone dicitur et est prime positionis nomen habens adiecta quae supradiximus; sicut et littera in nostra lingua adiecta habet: dicimus enim literatus, litterata, litteratum. Et diminitiuum gradum litteratura. Gramma igitur in greco, sephyr in ebreo. Grammatica litterata uel litteratura. Grammatus qui incipit initium litterarum ad legendum. Grammetus qui per semetipsum paginas aliorum bene potest legere. Grammaticus i. litterator uel litteratus, qui de litteris et de sillabis et reliquis supradictis sine ulla ambiguitate disputat.

$<2>$ Xenodochium locus uenerabilis in quo peregrini suscipiuntur. Pthochotropium locus uenerabilis in quo pauperes et infirmi homines pascuntur. Nosochomium locus uenerabilis in quo egroti curantur. Orfanotrophium locus uenerabilis in quo a parentibus orbati pueri pascuntur. Geronthochomium locus uenerabilis in quo pauperes et proter senectutem solam infirmi homines curantur. Brephothophium [sic] locus uenerabilis in quo infantes aluntur.

$<3>$ Glis glissis incrementum, glis glitis carduus, glis glidis terra tenax, glis gliris animal.

La première entrée porte sur les sens des termes gramma, grammatica, grammetus, grammatus et grammaticus. Les deux occurrences du verbe supradicere nous invitent à considérer qu'il s'agit d'un extrait d'un texte plus développé, peut-être un traité de grammaire ou un commentaire d'un grammairien. Je n'ai pas retrouvé la source de passage, mais on peut signaler qu'une partie de ce matériel se retrouve dans certaines grammaires carolingiennes ${ }^{83}$, dans des traités d'origine insulaire ${ }^{84}$ ou dans des glossaires ${ }^{85}$.

La seconde entrée est constituée d'un petit glossaire bien diffusé en France, en Allemagne, en Suisse et dans le nord de l'Italie. Il s'agit d'une liste de six termes grecs désignant des lieux de charité. Cette énumération est tirée à l'origine

83. Clemens Scottus, Ars grammatica, éd. J. Tolkiehn, Leipzig, Dieterich'sche Verlagsbuchhandlung (Philologus; Suppl. 20.3), 1928, p. 14, 1. 26-27: «Apud Hebraeos enim sephir uocatur, apud Graecos gramma, apud Latinos littera nominatur». Accessus à Priscien attribué à Létald de Micy copié dans Bern, BB, AA 90, fragm. 29, fol. 4v: «Grammatica autem diriuatur a gramma. Grammata uero a gramme, sicut Martianus docet: graece gramme linea dicitur, inde gramma littera, eo quod lineis formetur. Exinde uero grammatica est ars litteralis. Inde grammaticus litteratus" (in Anecdota Helvetica, éd. H. Hagen, Leipzig, Teubner, 1870, p. CLXviII, 1. 13-17).

84. Pseudo-Isidore, Liber de Numeris (München, BSB, Clm 14392, 74r) : «In tribus autem principalibus linguis littera sic appellatur: apud Hebreos sephyr, apud Grecos gramma, apud Latinos littera».

85. «Grammetus qui per paginas legere potest. Grammatus qui initium sumit legendi» («Excerpta ex codice Cassinensi 90", in Corpus Glossariorum Latinorum, V, éd. G. Goetz, Leipzig, Teubner, 1894, p. 569, 34-35). 
du Capitulaire d'Anségise de 827 et circule rapidement de façon indépendante à partir de la seconde moitié du IX $\mathrm{I}^{\mathrm{e}}$ siècle ${ }^{86}$, parfois dans des manuscrits d'auteurs classiques $^{87}$.

La dernière entrée propose une distinction entre quatre termes ayant tous la forme glis comme nominatif singulier: Glis glissis incrementum, glis glitis carduus, glis glidis terra tenax, glis gliris animal. Selon cette liste, glis glissis désigne un accroissement, glis glitis le chardon, tandis que glis glidis correspond à la terre glaise, et glis gliris à un animal. Ce terme ambigu apparaît régulièrement dans les textes grammaticaux tardo-antiques et carolingiens ${ }^{88}$. En partant de l'hypothèse que cette liste d'homonymes devait correspondre à une glose, j'ai recherché parmi ces textes liés à l'enseignement de la grammaire les occurrences de glis ou de glisco. Cette enquête a révélé la proximité de la liste du manuscrit de Juvénal avec plusieurs gloses carolingiennes sur Priscien et le commentaire de Remi d'Auxerre sur Eutychès ${ }^{89}$.

Glose carolingienne sur Priscien, Instit. VI, 248, 8-9 (à propos de glis gliris):

- Paris, BnF, lat. 7501, fol. 53v (IX ${ }^{3 / 3}$ pour le texte principal, IX-X pour les gloses, Corbie): "Glis gliris animal. Glis glisis incrementum. Glis glitis carduus. Glis glidis terra tenax vel tenera; glis gliris dicitur a gliscendo id est a crescendo. Crescit enim dormiendo. Glisco vero verbum venit a nomine glis glissis quod est incrementum»;

- Paris, BnF, lat. 7496, fol. 57v (IX ${ }^{3 / 4}$, Auxerre): «Genus muris quod nimio somnio dicitur crescere. Gliscere enim crescere. Glis glisis incrementum; glis uero glitis, carduus; glis glidis terra tenax»;

- München, BSB, Clm 18375, fol. 64v (IX ${ }^{\mathrm{ex}}$, ouest de l'Allemagne, peut-être Corvey?; textes et gloses proches du contenu de Paris, BnF, lat. $7501^{90}$ ) : "Glis gliris animal; glis glittis herba, lappa; glis glissis incrementum terre; glis glidis terra tenax»;

- Paris, BnF, lat. 7504, fol. 45r (IX ${ }^{3 / 3}$, région de la Loire): «Glis gliris animal, glis $g<l>i t i s$ genus herbe, glis glisis incrementum, quia gliscere crescere est»;

- Köln, Dombibl., 200, fol. 51r (IX ${ }^{2 / 3}$ pour le texte, IX-X pour les gloses, Prüm?): "glis gliris animal, glis glitis id est olus, glis glissis incrementum»;

86. Sur ce glossaire, voir KaCZYNSKI, 1983; Riou, 1990, p. 384, n. 47; OtTAVIANo, 2013-2014, p. 316317; Delmulle, 2016, p. 85-86.

87. Voir, par exemple, pour les manuscrits carolingiens de Virgile, OtTaviano, 2013-2014, P; p. 316-317.

88. On le rencontre, notamment, dans les grammaires de Charisius, Julien de Tolède, Priscien ou Clemens Scottus, dans le livre que Martianus Capella consacre à Grammatica, ou dans les commentaires sur Donat de Smaragde ou de Sedulius.

89. Mis à part pour le manuscrit de Rouen, les datations et localisations sont tirées des notices données dans Cinato, 2015. Je remercie profondément Franck Cinato de m'avoir communiqué ses notes sur cette glose et de m'avoir, notamment, signalé sa présence dans les manuscrits Paris, BnF, lat. 7496, Paris, BnF, lat. 10290 et Reims, Bibl. mun., 1094.

90. Cf. Cinato, 2015, p. 540. 
- Reims, Bibl. mun., 1094, fol. 132v (IX'1 pour le texte, IX $^{2}$ pour la glose, Soissons?): «glis gliris animal id est uermis; glis glisis terra tenax; glis glitis lappa» [les mots en romain sont ajoutés par une seconde main au début du $\mathrm{X}^{\mathrm{e}}$ siècle];

- Paris, BnF, lat. 7730, fol. 102v (IX ${ }^{4 / 4}$, Fleury ou Reims, glossae collectae sur l'Ars de Priscien): "glis glitis genus herbae, glis gilsis [sic] incrementum quia gliscere crescere est ${ }^{91}$;

- Paris, BnF, lat. 10290, fol. 67v (IX ${ }^{4 / 4}$ pour le texte principal, $\mathrm{X}^{\text {in }}$ pour les gloses qui nous intéressent, nord-est de la France, Paris ou Soissons?): «id est animal. Glis autem gliris herba, glis glisis incrementum» [main 1], «glis glitis carduus, glidis terra» [main 2].

Commentaire de Remi d'Auxerre sur Eutychès (à propos du verbe glisco dans le De verbo, 5, 449, 2):

- Paris, BnF, lat. 7499, fol. 73v ( $\mathrm{X}^{2}$, région de Corbie): «Glis, gliris animal est quod rata dicitur. Et glis glisis incrementum, id est aucmentum. Et glis, glitis carduus. Et glis, glidis, terra tenax, vel tenera. Glisco enim aut a nomine glis, gliris aut a glis, glissis venit id est incipio crescere»;

- Rouen, Bibl. mun., 1470, fol. 81v (XI, Fécamp): «Glires dicti sunt quia pingues eos facit somnum. Nam gliscere dicimus crescere. Hieme [Hiememe cod.] enim tota dormiunt, quasi mortui inmobiles permanent. Estiuo tempore reuiuiscunt. Glis glisis augmentum, crementum, glis, glitis graduus, glis glidis terra tenax».

En ce qui concerne Priscien, il est intéressant de noter que les gloses les plus proches de celle du manuscrit du Mont - les trois premières de ma liste - apparaissent dans trois des quatre exemplaires carolingiens transmettant l'ensemble du corpus priscianique ${ }^{92}$. Le quatrième représentant de cette famille est le manuscrit Paris, BnF, lat. 7504, qui ne présente que la moitié du matériel de la glose montoise. Le contenu du manuscrit Paris, BnF, lat. 10290 confirme qu'une partie de la tradition glossographique carolingienne sur Priscien ne renferme que les références à animal et à incrementum, ce qui a poussé un autre copiste à compléter la glose de ce manuscrit en ajoutant les définitions carduus et terra tenax. Même s'il conviendrait de poursuivre cette enquête en l'élargissant à d'autres corpus et à des manuscrits plus récents ${ }^{93}$, les sondages

91. La même glose se trouve aussi dans deux autres témoins de ces glossae collectae: Einsiedeln, Stiftsbibl. 32 ( $\mathrm{X}^{\text {in }}$, région du lac de Constance) et Leiden, UB, VLO 37 (IX ${ }^{\text {ex }}$, Reims?). Sur ce groupe de manuscrits, qui compte une branche alémanique et une branche française, voir CinATO, 2015, p. 378-379.

92. Cf. Cinato, 2015, p. 68-71.

93. On lit, par exemple, dans un commentaire auxerrois sur Phocas (Vaticano, BAV, Reg. lat. 1560, fol. 26r) : «Glis glitis lappa, glis gliris animal, glis glissis terra tenax [tenex p.c.]». Dans un manuscrit de Priscien copié en Allemagne au XII ${ }^{\mathrm{e}}$ siècle, on trouve la glose suivante: "Glis gliris animal, glis glisis incrementum terae, glis glitis carduus» (Erlangen, Universitätsbibl., 397, fol. 58v). Enfin, 
que j'ai pu faire soulignent que le contenu de la glose du Mont sur glis avec ses quatre significations est relativement rare. De ce point de vue, il est important de constater que les parallèles les plus proches se retrouvent dans des manuscrits provenant du nord-est de la France et liés à un petit nombre de centres, comme Soissons, Reims, Auxerre et Corbie.

\section{Conclusion}

L'analyse du contenu du manuscrit de Juvénal du Mont Saint-Michel nous permet d'émettre quelques hypothèses sur son origine. Le texte des Satires et ses gloses invitent à considérer que le modèle utilisé par les moines normands avait été copié dans le quart nord-est de la France durant le $\mathrm{X}^{\mathrm{e}}$ siècle, dans un centre appartenant au réseau d'écoles dans lequel était diffusé l'enseignement des maîtres auxerrois. L'étude des additions copiées par les mains A, B et D renvoie vers la même aire géographique et vers les mêmes milieux intellectuels et nous invite à considérer que les divers ajouts copiés après les Satires transmettent du matériel provenant sans doute du même centre que le reste du manuscrit. Malgré l'incertitude des localisations de manuscrits et le caractère extrêmement mouvant des gloses, plusieurs indices, parfois ténus, renvoient vers la province ecclésiastique de Reims.

Pour préciser davantage la région d'origine du matériel scolaire de ce manuscrit, il faudrait mener la même enquête sur l'ensemble des manuscrits copiés au Mont à la même époque par les mêmes personnes. Une étude comparée minutieuse du contenu de la dizaine de manuscrits copiés sous l'abbé Mainard II que nous avons conservés permettrait sans doute d'apporter quelques éclaircissements sur l'origine de la bibliothèque constituée par les premières générations de bénédictins au Mont. En effet, le manuscrit de Juvénal n'est pas le seul à refléter l'influence des écoles du nord-est de la France et notamment de la région de Reims. On trouve, par exemple, dans le manuscrit de Martianus Capella copié à la même époque non seulement le commentaire de Remi d'Auxerre sur le De Nuptiis, dont la diffusion semble liée initialement à Reims, mais aussi un poème de Gerbert de Reims composé avant 1002, probablement vers 996, c'est-à-dire juste quelques années avant la copie de ce volume montois (Avranches, Bibl. mun., 240, fol. 8r). Par ailleurs, Thierry Buquet a récemment découvert dans une reliure d'un manuscrit montois (Avranches, Bibl. mun., 97) un fragment du commentaire de Remi d'Auxerre sur l'Ars minor de Donat, dont la copie remonterait d'après lui au $\mathrm{XI}^{\mathrm{e}}$ siècle ou au début du siècle suivant mais qui pourrait être, selon moi, à peu près contemporaine de celle du manuscrit de Juvénal ${ }^{94}$.

le contenu de notre glose est proche de ce que l'on lit dans l'Elementarium de Papias (cité par Du Cange dans son Glossarium): "Glis glissis: incrementum; Glis glitis carduus; glis glitis terra tenax, glis glitis herba».

94. Thierry Buquet, «Un fragment de Rémi d'Auxerre dans la reliure d'un manuscrit du Mont Saint-Michel», dans Les Échos du Craham, 27/02/2019, [en ligne] https://craham.hypotheses. org/2012, ISSN : 2552-3139. Dans un commentaire à ce billet (posté le $1^{\text {er }}$ mars 2019), Stéphane 
Je me contenterai ici de proposer deux voies de transmission pouvant expliquer l'arrivée au Mont de ces commentaires auxerrois. L'enseignement diffusé dans les écoles du nord-est de la France a pu parvenir au Mont grâce à l'abbaye de Fleury-sur-Loire. Plusieurs liens sont attestés à partir de la fin du $\mathrm{X}^{\mathrm{e}}$ siècle entre Fleury et le Mont, notamment sous l'abbatiat de Mainard $\mathrm{II}^{95}$. Or, comme Fleury fut un des grands relais des gloses et commentaires auxerrois, il est tout à fait possible d'imaginer que c'est par l'intermédiaire de cette abbaye ligérienne que le texte de Juvénal et ses gloses auxerroises sont arrivés au Mont Saint-Michel vers l'an Mil. Mais il existe aussi une autre hypothèse, que j'aurais tendance à privilégier: les moines du Mont ont pu trouver leur manuscrit de Juvénal dans la région de Corbie. J. J. Alexander a, en effet, découvert que l'initiale de l'exemplaire montois des Satires de Perse (Paris, BnF, lat. 8055, p. 141), dans lequel on retrouve la main d'Hervardus, reproduisait une initiale très particulière provenant d'un psautier enluminé carolingien de Corbie (actuel Amiens, Bibl. mun., 18, fol. 31v) ${ }^{96}$. Cet indice extrêmement fort atteste l'existence d'échanges entre le Mont Saint-Michel et la grande bibliothèque de Corbie à l'époque de la copie du manuscrit de Juvénal et indique qu'un de ses copistes connaissait un volume conservé dans cet établissement de la province ecclésiastique de Reims. Il est donc fort possible que ce soit dans cette grande abbaye du nord de la France que les moines du Mont aient été chercher leurs modèles non seulement pour les Satires de Perse mais aussi pour celles de Juvénal.

Pour étayer cette hypothèse, il conviendrait d'étudier non seulement les autres manuscrits montois copiés à cette époque mais aussi l'influence que Corbie a pu exercer sur d'autres établissements normands en relation avec le Mont. Cette enquête devrait, notamment, se pencher sur Jumièges ${ }^{97}$ car plusieurs manuscrits carolingiens provenant de cette abbaye semblent avoir été copiés à Corbie ou dans le nord de la France ${ }^{98}$. On ignore l'époque à laquelle ces volumes arrivent en Normandie mais il est possible que Corbie ait fourni certains manuscrits aux moines de Jumièges lors de la restauration de cet établissement

Lecouteux rapproche l'écriture de ces fragments de celle d'Hervardus et propose une datation qui rejoint la mienne: «ces fragments proviennent donc probablement d'un manuscrit du Mont Saint-Michel copié entre la fin du $\mathrm{X}^{\mathrm{e}}$ et le premier quart du $\mathrm{XI}^{\mathrm{e}}$ siècle, donc de l'époque où d'autres commentaires de Remi d'Auxerre furent copiés au Mont Saint-Michel (commentaires sur Juvénal, Martianus Capella, etc.)».

95. La main principale du manuscrit de Juvénal a, par exemple, copié une liste des moines du Mont sous l'abbé Mainard II (996-1009) dans l'actuel Orléans, Bibl. mun., 105, un manuscrit d'origine anglaise arrivé à Fleury probablement sous l'abbatiat d'Abbon (988-1004). Par ailleurs, deux manuscrits copiés dans la région d'Orléans arrivent au Mont Saint-Michel au cours du Moyen Âge: Avranches, Bibl. mun., 32 (IX-X, provenant de Saint-Mesmin de Micy) et Avranches, Bibl. mun., 238 ( $\mathrm{IX}^{2 / 3}$, copié dans la région de la Loire, ce manuscrit est sans doute déjà au Mont au XI ${ }^{\mathrm{e}}$ siècle).

96. AleXANDER, 1967

97. Jumièges faisait partie des établissements liés au Mont avant même la mise en place du réseau spirituel volpianien, cf. LeCouteux, 2015, I, p. 178-180.

98. Il s'agit des manuscrits 141, 147, 423, 496 et 557 (feuillets de garde) de la bibliothèque municipale de Rouen. Je remercie Jérémy Delmulle d'avoir attiré mon attention sur ce groupe de manuscrits. 
durant le deuxième quart du $\mathrm{X}^{\mathrm{e}}$ siècle et, dans cette perspective il est tentant d'imaginer qu'elle ait pu jouer un rôle similaire, peut-être justement grâce à ses liens avec Jumièges ${ }^{99}$, lors de la reprise en main du Mont par les bénédictins quelques décennies plus tard.

\section{Bibliographie}

Alexander, Jonathan J. G., «A Romanesque Copy from Mont Saint-Michel of an Initial in the Corbie Psalter», in Millénaire monastique du Mont Saint-Michel, t. 2, Vie montoise et rayonnement intellectuel, Raymonde Foreville (dir.), Paris, Lethielleux (Bibliothèque d'histoire et d'archéologie chrétiennes), 1967, p. 239-244.

Alexander, Jonathan J. G., Norman Illumination at Mont St Michel. 966-110o, Oxford, Clarendon Press, 1970.

AvriL, François, «La décoration des manuscrits au Mont Saint-Michel», in Millénaire monastique du Mont Saint-Michel, t. 2, Vie montoise et rayonnement intellectuel, Raymonde Foreville (dir.), Paris, Lethielleux (Bibliothèque d'histoire et d'archéologie chrétiennes), 1967, p. 203-238.

BeEson, Charles H., "The Authorship of Quid sit Ceroma», in Classical and Medieval Studies in Honor of Edward Kennard Rand, L. W. Jones (éd.), New York, 1938, p. 1-7.

BISCHOFF, Bernhard, Katalog der festländischen Handschriften des neunten Jahrhunderts, t. II, Wiesbaden, Harrassowitz, 2004.

BIsCHOFF, Bernhard, Katalog der festländischen Handschriften des neunten Jahrhunderts, t. III, Wiesbaden, Harrassowitz, 2014.

Buquet, Thierry, «Un fragment de Rémi d'Auxerre dans la reliure d'un manuscrit du Mont Saint-Michel», dans Les Échos du Craham, 27/02/2019, [en ligne] https:// craham.hypotheses.org/2012, ISSN : 2552-3139.

Büren, Veronika (von), «Heiricus Autissiodorensis mon.», in Clavis Scriptorum Latinorum Medii Aevi. Auctores Galliae, III, Marie-Hélène Jullien (dir.), Turnhout, Brepols (Corpus christianorum continuatio mediaevalis), 2010a, p. 375-405.

Büren, Veronika (von), «Le Juvénal des Carolingiens à la lumière du Ms Cambridge King's College 52 », Antiquité tardive, 18, 2010b, p. 115-137.

Cinato, Franck, L'Ars grammatica de Priscien vue à travers les gloses carolingiennes, Turnhout, Brepols (Studia artistarum), 2015.

Cleaver, Laura, "The Monastic Library at Le Bec», in A Companion to the Abbey of Le Bec in the Central Middle Ages (11th-13th Centuries), Benjamin PoHL, Laura L. Gathagan (éd.), Leiden, Brill (Brill's companions to European history, 13), 2018, p. 171-295.

Contreni, John J., Codex Laudunensis 468. A ninth-century guide to Virgil, Sedulius, and the liberal arts, Turnhout, Brepols (Armarium codicum insignium, 3), 1984.

99. De ce point de vue, la présence de la Genealogia Enee à Jumièges pourrait être bien antérieure à la copie de Rouen, Bibl. mun., 1177. 
Delisle, Léopold, Chronique de Robert de Torigni, abbé du Mont-Saint-Michel suivie de divers opuscules historiques de cet auteur et de plusieurs religieux de la même abbaye, II, Rouen, Le Brument (Société de l'histoire de Normandie), 1873.

Delmulle, Jérémy «Sur une acception médiévale du lat. locusta désignant une herbe sauvage comestible», Archivum Latinitatis Medii Aevi, 74, 2016, p. 67-88.

Dumville, David N. «An Early Text of Geoffrey of Monmouth's Historia Regum Britanniae and the Circulation of some Latin Histories in Twelfth-Century Normandy», Arthurian Literature, 4, 1985, p. 1-36.

Duplessis, Frédéric, "Les "proto-accessus" carolingiens sur Juvénal: formation et diffusion ", Archivum Latinitatis Medii Aevi, 75, 2017, p. 107-148.

Duplessis, Frédéric, «Diffusion et réception médiévales des épigrammes d’Ausone consacrées à Diogène", in La réception d'Ausone dans les littératures européennes, Étienne WolfF (dir.), Bordeaux, Ausonius (Scripta Receptoria, 15), 2019, p. 67-83.

Duplessis, Frédéric, «Langue et littérature latines du Moyen Âge», Annuaire de l'École pratique des hautes études (EPHE), Section des sciences historiques et philologiques, 151, 2020, p. 195-209.

GrazzINI, Stefano, Scholia in Iuuenalem recentiora secundum recensiones $\varphi$ et $\chi, 1$, Pise, Edizioni della Normale (Testi e commenti, 11), 2011.

GrazzINI, Stefano, Scholia in Iuuenalem recentiora secundum recensiones $\varphi$ et $\chi, 2$, Pise, Edizioni della Normale (Testi e commenti, 21), 2018.

Jeudy, Colette, «Donat et commentateurs de Donat à l'abbaye de Ripoll au X $\mathrm{X}^{\mathrm{e}}$ siècle (ms. Barcelone, Archivo de la Corona de Aragón, Ripoll 46) », in Lettres latines du Moyen Âge et de la Renaissance, Guy Cambier, Carl Deroux, Jean Préaux (éd.), Bruxelles (Latomus; 158), 1978, p. 56-75.

Jeudy, Colette, «Remigii Autissiodorensis opera (Clavis)», in L'École carolingienne d'Auxerre de Murethach à Remi, 830-908 (Entretiens d'Auxerre, 1989), Dominique Iogna-Prat, Colette Jeudy, Guy Lobrichon (éd.), Paris, Beauchesne (L'Histoire dans l'actualité), 1991, p. 457-500.

Kaczynski, Bernice M. «Some St. Gall Glosses on Greek Philanthropic Nomenclature», Speculum, 58-4, 1983, p. 1008-1017.

Knoche, Ulrich, Handschriftliche Grundlagen des Juvenaltextes, Leipzig, Dieterich'sche Verlagsbuchhandlung (Philologus, Suppl. 33.1), 1940.

Law, Vivien, Wisdom, Authority and Grammar in the Seventh Century. Decoding Virgilius Maro Grammaticus, Cambridge, Cambridge University Press, 1995.

Lecouteux, Stéphane, Réseaux de confraternité et histoire des bibliothèques. L'exemple de l'abbaye bénédictine de La Trinité de Fécamp, thèse de doctorat en Histoire médiévale, Caen, UFR Histoire (dir. Catherine Jacquemard et Anne-Marie TurcanVerkerk), 2015, 2 vol., HAL Id: tel-01572371.

Lendinara, Patrizia, «The Scholica Graecarum glossarum and Martianus Capella», in Carolingian Scholarship and Martianus Capella. Ninth-Century Commentary Traditions on "De nuptiis" in Context, Mariken Teeuwen, Sinéad O'Sullivan (éd.), Turnhout, Brepols (Cultural Encounters in Late Antiquity and the Middle Ages, 12), 2011, p. 301-361.

Munk Olsen, Birger, L'étude des auteurs classiques latins aux XI ${ }^{e}$ et XII siècles, t. II, Paris, Éditions du CNRS (Documents, études et répertoires), 1985. 
Munk OlsEn, Birger, L'étude des auteurs classiques latins aux XI et XII e siècles, t. IV-1, La réception de la littérature classique, travaux philologiques, Paris, CNRS Éditions (Documents, études et répertoires), 2009.

OrLANDI, Giovanni, «Lupus Ferrariensis abb.», in La trasmissione dei testi latini del Medioevo. Mediaeval Latin Texts and Their Transmission, t. 3, Paolo Chiesa, Lucia CASTAldi (dir.), Florence, SISMEL Edizioni del Galluzzo (Millennio medievale), 2008, p. 432-454.

Ottaviano, Silvia, La tradizione delle opere di Virgilio tra IX e XI sec., Diploma di Perfezionamento in Discipline letterarie, filologiche e storiche classiche, Scuola Normale superiore di Pisa, 2013-2014.

PARETTI, Luca, «Lettere erudite di Sedulio Scoto?», Rationes Rerum, 2, 2013, p. 195-216.

PezÉ, Warren, «Débat doctrinal et genre littéraire à l'époque carolingienne: les opuscules théologiques de Gottschalk d'Orbais », Revue de l'histoire des religions, 2017-1, p. 25-72, DOI: $10.4000 /$ rhr.8653.

Riou, Yves-François, "Codicologie et notation neumatiques (suite et fin)», Cahiers de civilisation médiévale, $33^{\mathrm{e}}$ année ( $\mathrm{n}^{\mathrm{o}}$ 132), Octobre-décembre 1990, p. 381-396.

SANDFORD, Eva M., «Bread and Circuses», Classical Weekly, 45, 1951, p. 17-21.

SAVAGE, John, «The Manuscripts of Servius's Commentary on Virgil», Harvard Studies in Classical Philology, 45, 1934, p. 157-204.

Sims-Williams, Patrick, Religion and Literature in Western England, 6oo-80o, Cambridge, Cambridge University Press (Cambridge Studies in Anglo-Saxon England, 3), 1990.

Tarrant, Richard J., "Juvenal», in Texts and Transmission. A Survey of the Latin Classics, Leighton D. Reynolds (éd.), Oxford, Clarendon Press, 1983, p. 200-203.

Traube, Ludwig, "Computus Helperici», Neues Archiv der Gesellschaft für ältere deutsche Geschichtskunde, XVIII, 1893, p. 71-105 et p. 724-725. 


\section{Annexe: édition de la Genealogia Enee et Priami}

Le manuscrit de base pour l'établissement du texte est le manuscrit de Paris. Pour les phrases 6-15, mon édition s'appuie aussi sur le chapitre $132 \mathrm{du}$ premier Mythographe du Vatican et sur l'Origo Troianorum éditée par S. Ottaviano à partir de quatre témoins.

L'apparat ne tient pas compte des variantes orthographiques minimes concernant les noms propres (comme Aeneas/Eneas, Erichtonius/Erictonius, Iuppiter/Iupiter ou Asaracus / Assaracus).

- Montep.: Montpellier, BIUM, H 253, f. 12or

- Paris.: Paris, BNF, lat. 8070, f. 126r-127r

- Rotom.: Rouen, BM, 1177 [U.74], f. 289rv

- Orig.: «Origo Troianorum», éd. Ottaviano, La tradizione delle opere di Virgilio, p. 304 (colonne 1)

- Myth.: Vaticano, BAV, Reg. lat. 1401, f. 2 or (cf. Mythographus Vaticanus I, cap. 132, éd. Kulcsár, p. 55, 1. 5-16

$<$ Genealogia Enee et Priami >

(1) Sic ordinatur genealogia Eneae et Priami qui expugnatus est in Troia:

(2) Iuppiter uir magni ingenii in Creta fuit insula et habuit duas uxores id est Maiam et Electram filias Athlantis a quo mons Athlas nominatur. (3) Et genuit Iuppiter Mercurium ex Maia et Dardanum ex Electra. (4) Mercurius itaque ipse est origo Grecorum omnium. (5) Dardanus uero origo Troianorum et apud Iouem maior fuit amor Maiae et filii. (6) Dardanus uero ex responso deorum locum mutans ab Italia per Traciam Samo delatus est quam Samotraciam nominauit. (7) Exhinc ad Frigiam uenit quam Dardaniam de suo nomine nominauit. (8) Ex quo natus est Ericthonius qui hisdem locis regnauit. (9) Et ex Erictonio Tros, qui iustitia et pietate laudabilis fuit; isque ut memoriam sui nominis faceret eternam Troiam apellari iussit. (10) Tros autem duos habuit filios, Ilum et Assaracum, a quo Ilo Ilium dictum est, id est Troia. (11) Ilo autem Laomedon filius fuit. (12) Ex Laomedonte Priamus natus est qui expugnatus in Troia. (13) Assaracus uero genuit Capin. (14) Capis Anchisen. (15) Anchises autem Enean procreauit. (16) Genealogia utrorumque ita retrorsum reuertitur: Priamus filius Laomedontis filii Ili filii Trois filii Erictonii filii Dardani filii Iouis filii Saturni filii Celi. (17) Item Eneas filius Anchise filii Capin filii Asaraci filii Trois filii Erictonii filii Dardani filii Iouis filii Saturni filii Celi.

(1) sic - troia] quomodo coniungitur genealogia aeneae et priami qui expugnatus est in troia sic ordinatur Montep. (2) iuppiter] iouis Montep. iupiter saturni filius celii filius Rotom. | habuit duas] duas habuit Montep. | id est maiam] maiam scilicet Rotom. | filias athlantis] athlantis filias Rotom. (3) et] om. Rotom.| iupiter] iouis Montep.Paris. (4) est - omnium] omnium grecorum est origo Rotom. (5) fuit add. Rotom. post troianorum | et - amor] fuit autem apud iouem amor maior Rotom. | apud] caput Paris. (6) dardanus 
- italia] dardanus ex ioue et electra filia atlantis natus ab italia ex responso locum cummutans Orig. idem dardanus ab italie regione tuscia ex responso locum mutans Myth. | uero] namque Rotom. | delatus est] dilatus est Paris.Rotom. | est om. Montep. (7) om. Rotom. | exhinc] et hinc Myth. | uenit] deuenit Orig.Myth. | de] a Orig.Myth. (8) hisdem] in hisdem Orig. in iisdem corr. Ottaviano | in istis Myth. | hisdem - regnauit] isdem regnauit locis Rotom. (9) et ex] ex Orig.Myth. | iustitia] in iusticia Rotom. | ut] uit Paris. | sui nominis] nominis sui Rotom. | troiam apellari] urbem troiam suo nomine appellari Rotom. | apellari iussit] nominauit Myth. (10) tros autem] tros Rotom. et ipse Orig. qui etiam tros Myth. | duos habuit filios] duos filios habuit Montep.Rotom. habuit duos filios Orig. filios habuit Myth. | ilum] id est ilum Montep. | et assaracum] asaracumque Montep.Myth. a - troia] is ilus qui maior natu erat regnauit atque ilium condidit ciuitatem Orig. hic quia maior natu erat regnauit atque troiam de suo nomine ilium nominauit assaricus a primatu recessit Myth.| ilo] ilio Rotom. (11-12) genuitque laomedontem de quo natus est priamus Orig. (11) ilo] ili Paris.Rotom. ilus corr. Kulcsár| autem] om. Myth. | laomedon] hic laomedon Rotom. | filius fuit] filium habuit Myth. (12) priamus] primus priamus Myth. | natus est] fuit natus Montep. | qui - troia] om. Myth. [ expugnatus] est add. Montep.Rotom. (13) uero] om. Paris.Myth. | genuit capin] capin genuit Myth. | capin] capis Rotom. (14) capis anchisen] ex quo natus est anchises Montep. ex quo anchises Orig. ex quo anchises editus est Myth. (15) anchises - procreauit] ipse enim genuit aeneam Orig. | anchises autem] anchisesque Myth. | autem] om. Rotom. | eneas aschanium aschanius siluium siluius brutum a quo britones dicuntur et originem ducunt post procreauit add. Rotom. (16) utrorumque] utrorum Paris. | reuertitur] reuoluitur Rotom. (17) eneas filius anchise] brutus filius siluii filii aschanii filii enee filii anchise Rotom. 